\title{
Assessment of the accuracy of the conventional ray-tracing technique: Implications in remote sensing and radiative transfer involving ice clouds
}

\author{
Lei Bi ${ }^{a}$,* , Ping Yang ${ }^{a}$, Chao Liu ${ }^{a}$, Bingqi Yi ${ }^{a}$, Bryan A. Baum ${ }^{b}$, \\ Bastiaan van Diedenhoven ${ }^{\mathrm{c}}$, Hironobu Iwabuchi ${ }^{\mathrm{d}}$ \\ a Department of Atmospheric Sciences, Texas AE'M University, TAMU 3150, College Station, TX 77843-3150, USA \\ b Space Science and Engineering Center, University of Wisconsin-Madison, 1225 West Dayton St., USA \\ c Columbia University, Center for Climate System Research, NASA Goddard Institute for Space Studies, 2880 Broadway, \\ New York, NY 10025, USA \\ ${ }^{\mathrm{d}}$ Graduate School of Science, Tohoku University, Sendai, Japan
}

\section{A R T I C L E I N F O}

\section{Article history:}

Received 6 November 2013

Received in revised form

17 March 2014

Accepted 18 March 2014

Available online 26 March 2014

Keywords:

Ice clouds

Light scattering

Remote sensing

Radiative transfer

\begin{abstract}
A B S T R A C T
A fundamental problem in remote sensing and radiative transfer simulations involving ice clouds is the ability to compute accurate optical properties for individual ice particles. While relatively simple and intuitively appealing, the conventional geometric-optics method (CGOM) is used frequently for the solution of light scattering by ice crystals. Due to the approximations in the ray-tracing technique, the CGOM accuracy is not well quantified. The result is that the uncertainties are introduced that can impact many applications. Improvements in the Invariant Imbedding T-matrix method (II-TM) and the Improved GeometricOptics Method (IGOM) provide a mechanism to assess the aforementioned uncertainties. The results computed by the II-TM+IGOM are considered as a benchmark because the IITM solves Maxwell's equations from first principles and is applicable to particle size parameters ranging into the domain at which the IGOM has reasonable accuracy. To assess the uncertainties with the CGOM in remote sensing and radiative transfer simulations, two independent optical property datasets of hexagonal columns are developed for sensitivity studies by using the CGOM and the II-TM+IGOM, respectively. Ice cloud bulk optical properties obtained from the two datasets are compared and subsequently applied to retrieve the optical thickness and effective diameter from Moderate Resolution Imaging Spectroradiometer (MODIS) measurements. Additionally, the bulk optical properties are tested in broadband radiative transfer (RT) simulations using the general circulation model (GCM) version of the Rapid Radiative Transfer Model (RRTMG) that is adopted in the National Center for Atmospheric Research (NCAR) Community Atmosphere Model (CAM, version 5.1). For MODIS retrievals, the mean bias of uncertainties of applying the CGOM in shortwave bands $(0.86$ and $2.13 \mu \mathrm{m})$ can be up to $5 \%$ in the optical thickness and as high as $20 \%$ in the effective diameter, depending on cloud optical thickness and effective diameter. In the MODIS infrared window bands centered at $8.5,11$, and $12 \mu \mathrm{m}$, biases in the optical thickness and effective diameter are up to $12 \%$ and $10 \%$, respectively. The CGOM-based simulation errors in ice cloud radiative forcing calculations are on the order of $10 \mathrm{~W} \mathrm{~m}^{-2}$. (c) 2014 Elsevier Ltd. All rights reserved.
\end{abstract}

\footnotetext{
* Corresponding author.

E-mail address: bilei@tamu.edu (L. Bi).
} 


\section{Introduction}

Ice clouds are known to play an important role in regulating atmospheric radiation through interaction with both solar and infrared (IR) radiation fields [1,2]. Theoretical modeling of ice cloud radiative effects is indispensable in many atmospheric applications. The modeling approach typically begins with the single scattering of light by individual ice particles of different habits (shapes) and sizes contained in a scattering volume element, followed by the multiple-scattering calculations (i.e., the radiative transfer, or RT) involving ice clouds. For example, inferring the microphysical properties of ice clouds from observations by ground- and satellite-based instruments requires comparing the instrument observations to the model-simulated results obtained from the singlescattering properties and relevant multiple-scattering simulations [3-7]. To understand the ice cloud impact in the climate models, an accurate representation of ice cloud bulk-scattering properties is a critical component required by RT models to quantify the cloud radiative effect (CRE), such as radiative forcing of natural cirrus clouds $[8,9]$ and anthropogenic contrails $[10,11]$. For this reason, much effort has been dedicated to developing physically representative ice cloud models (e.g., ice habits, particle size distributions, and mass-dimension relationships) and accurately computing the single-scattering properties of individual ice particles (i.e., the extinction efficiency, the single-scattering albedo, and the phase matrix).

However, solving Maxwell's equations for light scattering by ice particles from first principles is much more complex than light scattering by water droplets. Ice particles are nonspherical and morphologically complex, while having a broad range of particle sizes ranging from a few microns to centimeters. In a historical context, the majority of simulations [12-19] are based on geometricoptics principles that are asymptotically correct and approximately valid at large size parameters, i.e., when the particle size is sufficiently large relative to the wavelength. The ratio of the particle circumference to the wavelength is known as the size parameter. A number of physical-geometric optics methods have also been proposed to improve the accuracy in geometric-optics approximations [20-26].

Deschamps [27] addressed the importance of the "ray" concept of light in engineering, stating that "if the nature of light and Maxwell's equations had been known, earlier optical instruments would not have been invented so readily"! Similarly, the application of ray techniques in solving light scattering by individual ice particles in the atmospheric radiation discipline plays a critical role because it provides a first-order approximation for higher frequency scattering where rigorous solutions are unavailable. In other words, the knowledge of ice cloud radiation would have been severely limited in a modeling perspective without employing the geometric-optics method. A more specific example is that for an ice particle with a size parameter larger than 200, the ability to obtain a rigorous set of optical properties is almost beyond our current first-principle modeling capabilities. For this reason, the geometric-optics methods continue to be popular, but there is a growing awareness of the need to provide an accuracy assessment due to the inherent approximations [e.g.,28,29].

With the development of computational electrodynamics, numerical techniques to solve Maxwell's equations are now available to obtain numerically exact solutions. The power of these numerical techniques began to have an impact within the past two decades. A few frequently used computational techniques are the finitedifference time-domain (FDTD) [30-33], the pseudospectral time-domain (PSTD) [34-36], the discretedipole-approximation (DDA) [37-40], and the T-matrix method [41-45]. Based on the numerical techniques, a much better knowledge has been obtained of the optical properties of particles at small-to-moderate size parameters. The applicability of the geometric-optics in different size parameter domains is investigated whenever possible. For example, Mishchenko and Macke [46] investigated T-matrix computations of light scattering by circular cylinders and found that ice halos, an optical phenomenon predicted from geometric optics, would not be observed when the particle size parameter is less than approximately 100 . Unlike the geometric-optics methods, the performance of numerical methods strongly depends on computational resources. As computer power increases, some new domains of rigorous solutions are being conquered. Meanwhile, because of its simplicity, the use of the geometric-optics method is preferred for a complete range of ice particle sizes in the wavelength spectrum from UV to the near-infrared.

To our knowledge, the induced uncertainties due to the nature of the approximations inherent in the ray-tracing technique have not yet been assessed in remote sensing and climate studies involving ice clouds, most likely because the rigorous solution domain is still extremely limited. The Invariant Imbedding T-matrix method (II-TM) [47-50] is applicable to a broad range of size parameters from the Rayleigh region up to the geometric optics domain where ice halos are observed. In this study, we use the II-TM to assess the accuracy of geometric-optics approximation and the resulting uncertainties in remote sensing and radiative transfer simulations.

The theoretical components of the employed geometricoptics method are delineated before the assessment because a number of modifications in geometric-optics methods exist in the literature. A brief review of available geometric-optics methods is provided in $\mathrm{Bi}$ and Yang [51]. The geometric optics methods most frequently used are the conventional geometric-optics method (CGOM) and the improved geometric-optics method (IGOM), although a few more rigorous, but relatively less computationally efficient, methods have also been developed [20-26]. One unique difference between the CGOM and the IGOM is that the latter considers the spreading of scattered beams (a physical-optics effect) when propagating from the near-field to the far-field region. As the particle size parameter increases, the IGOM simulated phase matrix transitions to that from the CGOM when the spreading effect is negligible. With the ray-spreading effect incorporated, the IGOM is applicable to relatively small size parameters (20-100) where the ice halos disappear, but the geometric-optics principles are still more 
or less valid in estimating the internal electromagnetic field. The CGOM and the IGOM also differ in the computation of the extinction efficiency $Q_{\text {ext }}$, defined by the ratio of particle extinction cross section to particle projected area. The CGOM empirically assumes that the extinction efficiency is constant at a value of 2 in conjunction with the blocking effect and the diffraction effect whereas the IGOM computes the extinction efficiency based on a physical-optics approximation that takes into account the interference between the diffraction and the forward scattered rays.

In this study, we begin with the assessment of the accuracy of the single-scattering properties derived from the CGOM and the IGOM in the T-matrix applicable domains. Next, we use a combination of the II-TM and the IGOM for computing the single-scattering properties in a complete range of particle sizes ranging from 2 to 10,000 um in maximum diameter. Based on the finding that the IGOM and the T-matrix results are very similar at the transition regions from wave optics to geometric optics, we consider the II-TM+IGOM results as a benchmark and assess the uncertainties of applying the CGOM to an entire range of particle size parameters. From an application perspective, we then assess the uncertainty of using the CGOM in retrieving the ice particle effective diameter and optical thickness from Moderate Resolution Imaging Spectroradiometer (MODIS) measurements and the broadband radiative forcing calculations using the general circulation model (GCM) version of the Rapid Radiative Transfer Model (RRTMG), which is the model employed in the Community Atmosphere Model (CAM, version 5.1) developed at National Center for Atmospheric Research. For simplicity, the ice particles are assumed to be smooth-faceted hexagonal columns. Although atmospheric ice habits are diverse, the accuracy of the CGOM, IGOM, and II-TM is not assumed to change for different habits.

The remainder of this paper is organized as follows. In Section 2, we describe the numerical methods used for computing the optical properties of ice particles. The numerical results for the optical properties of hexagonal ice particles are shown in Section 3, including the comparison of the single-scattering properties and bulk-scattering properties computed from the CGOM and the II-TM+IGOM. Section 4 provides sensitivity studies to assess the uncertainties of applying the CGOM in the applications of remote-sensing retrievals. In Section 5, the uncertainties in radiative forcing calculations due to applying the CGOM are quantified through a single-column radiative transfer model and a GCM. The conclusions of this study are given in Section 6.

\section{Computational methods}

In this section, we briefly outline the principles of the computational methods of the CGOM, the IGOM, and the II-TM. The readers are referred to $[51,52]$ as primary references for the applications of geometric optics. A common theoretical basis of the CGOM is a combination of Fraunhofer diffraction by the particle shadow (Fig. 1a),
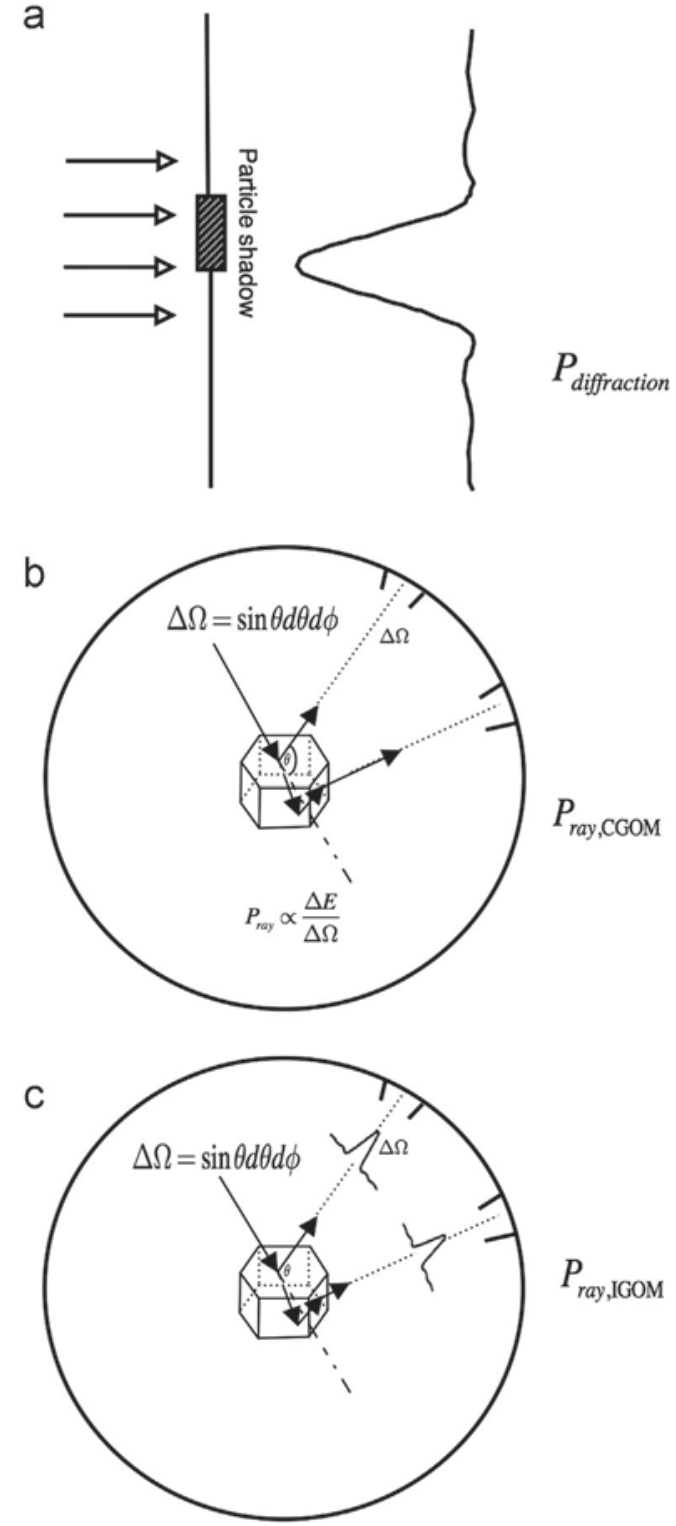

Fig. 1. Conceptual figure of geometric-optics principles. $P_{\text {diffaction }}$ indicates the phase function associated with Fraunhofer diffraction. $P_{\text {ray, CGom }}$ and $P_{\text {ray.IGom }}$ are phase functions associated with the scattered rays computed from the CGOM and the IGOM, respectively. In the CGOM, the phase function $P_{\text {ray }}$ is computed from the division of energy $\Delta E$ with respect to the differential solid angle $\Delta \Omega$.

and the angular distribution of scattered intensity obtained from the ray-tracing technique shown in Fig. 1b. Specifically, the scattering phase function from the ray-tracing process is obtained from the division of the energy received by each detector and the solid angle. The extinction efficiency is assumed to be 2 and arises separately from the diffraction and the ray-tracing part. The absorption efficiency is computed based on the energy lost in the ray-tracing process. The assumption of a constant value of $Q_{\text {ext }}$ in the CGOM may affect the accuracy of the single-scattering albedo, $1-Q_{a b s} / Q_{e x t}$, where $Q_{s c a}$ and $Q_{a b s}$ are the scattering and absorption efficiencies, respectively. 
The largest advantage of the CGOM is in its simplicity and flexibility to adapt to particle geometries; however, it suffers from several drawbacks. The solid angle is zero in both the forward and backscattering directions, thus causing a singularity problem. In most CGOM algorithms, values of the phase function in the direct forward and backward directions are obtained through extrapolation of the near-forward and near-backward directions. Moreover, if the particle is in a fixed orientation, the phase function is not a continuous curve but several discrete points. Although the random-orientation condition produces a relatively smooth phase function, an isolated peak is usually observed in the direction of forward scattering, commonly called the delta-transmission [15] energy, that is fundamentally related to parallel facets in a hexagonal ice habit. In the case of distorted or roughened particles, the delta-transmission term is not observed [53]. Mishchenko and Macke [54] specifically explained that the delta-transmission term exists because of the missing physical-optics effect. When the particle is non-absorptive, the angular distribution of scattered energy is evidently size-independent, but this is contradictory to the wave

Table 1

Theoretical components involved in the CGOM and the IGOM.

\begin{tabular}{lll}
\hline & CGOM & IGOM \\
\hline Extinction efficiency & 2 & Asymptotic value is 2 \\
Delta transmission & Removed & Removed \\
Ray spreading & No & Yes \\
Inhomogeneous wave & Yes & Yes \\
\hline
\end{tabular}

theory of light. Furthermore, the extinction efficiency is an asymptotic value and does not capture the sizedependence for small and moderate size parameters.

To overcome the shortcomings of the CGOM, geometric-optics principles are employed to compute either the internal field or the particle surface field, and the far field is obtained through exact electromagnetic volume integral equations or surface integral equations [20-26]. Within the new framework, the physical-optics effects are taken into account. For example, the extinction efficiency considers the interference between the diffraction and the waves associated with the forward scattering rays. The diffraction effect associated with outgoing scattered beams (Fig. 1c) is taken into account so that the scattering phase function from the ray-tracing calculation is size-dependent. For example, halos will only appear when the particle size parameter is sufficiently large (more than 100), where the ray-spreading effect is small and the rays are strongly localized. Yang and Liou [21] proposed a simplified algorithm to account for the physical-optics effect in the phase matrix computation that is implemented in IGOM.

When ice particles are absorptive, most localized waves inside the particle are inhomogeneous. In this study, we have considered the inhomogeneous nature of localized waves in the ray-tracing process in both the CGOM and the IGOM, although for simplicity the effect is usually neglected in many studies. Two obvious reasons to justify the consideration of inhomogeneous waves are that: (1) the energy is not conserved at the first-order of refraction if the inhomogeneity of localized waves is omitted; (2) the asymptotic value of the extinction efficiency derived from
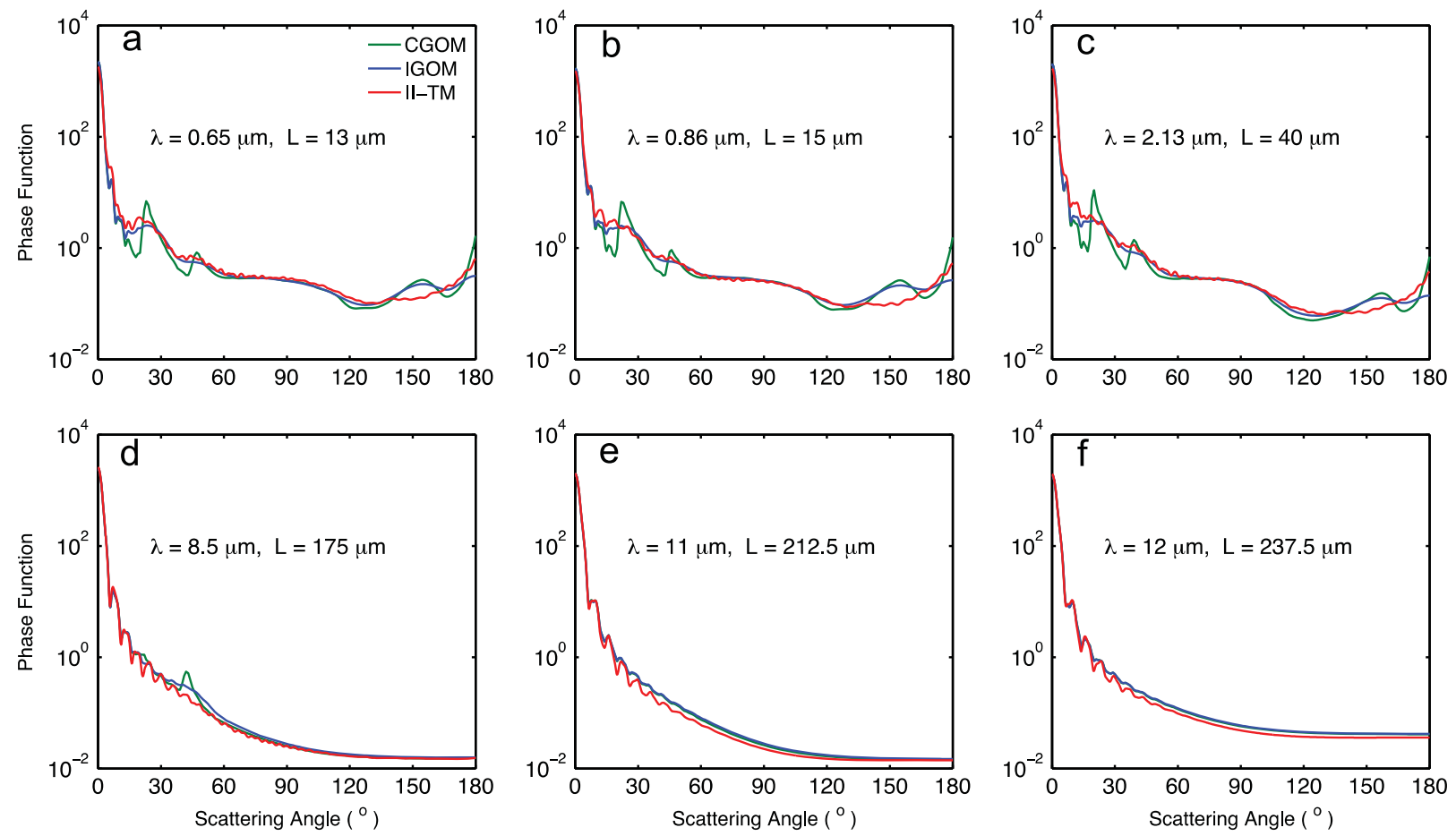

Fig. 2. Comparison of phase functions computed from the II-TM, the IGOM and the CGOM. The wavelength and the hexagonal column height are indicated in each sub-figure. The size parameters for each sub-figure from (a)-(f) are approximately 126, 110, 118, 129,121 and 124. 
the IGOM differs from 2 by a non-negligible amount if not considering the electric field component along the propagation direction.

In consideration of a number of geometric-optics algorithms, we specifically delineate the theoretical components involved in the CGOM and the IGOM in Table 1. Note that the delta-transmission peak has been removed in the CGOM by considering the physical-optics effect.

For a particle having a small size parameter, where the ray-concept is inappropriate for calculating the electromagnetic field inside the particle or particle surface, the IGOM gives inaccurate results; Maxwell's equations need to be solved. The transition (or T-) matrix method in conjunction with the extended-boundary condition (EBC) technique in the T-matrix computations pioneered by Waterman [41,42] is a powerful approach for computing the optical properties of nonspherical particles. In principle, the EBC is applicable to an arbitrarily shaped nonspherical particle; however, the EBC is most successful in cases of axially symmetric particles. For example, for a hexagonal particle whose geometry is of 6-fold rotational symmetry, the EBC's applicable size parameter region is substantially narrowed in comparison to spheroid cases.
Note that in the EBC method, the T-matrix is computed from surface integrals over the particle surface. Alternatively, the T-matrix can be derived from an electromagnetic volume integral equation and computed from an invariant imbedding procedure. One unique advantage of the invariant imbedding approach is that the algorithm is stable and flexible in adapting to a given particle geometry. We have extended the II-TM applicable region to large size parameters for nonspherical particles [47-49]. Here, we will not discuss the theoretical basis of the II-TM as the details can be found in [47-50].

\section{Optical properties of ice particles}

The optical properties of hexagonal ice particles are computed in a spectral region from 0.2 to $100 \mu \mathrm{m}$. The aspect ratio of hexagonal ice particles is the same as the geometry defined in [55] (their Table 1). Six wavelengths in the visible and infrared are selected for remote-sensing studies: $0.65,0.86,2.13,8.5,11$, and $12 \mu \mathrm{m}$. The ice refractive indices compiled by Warren and Brandt [56] are used in the simulations.
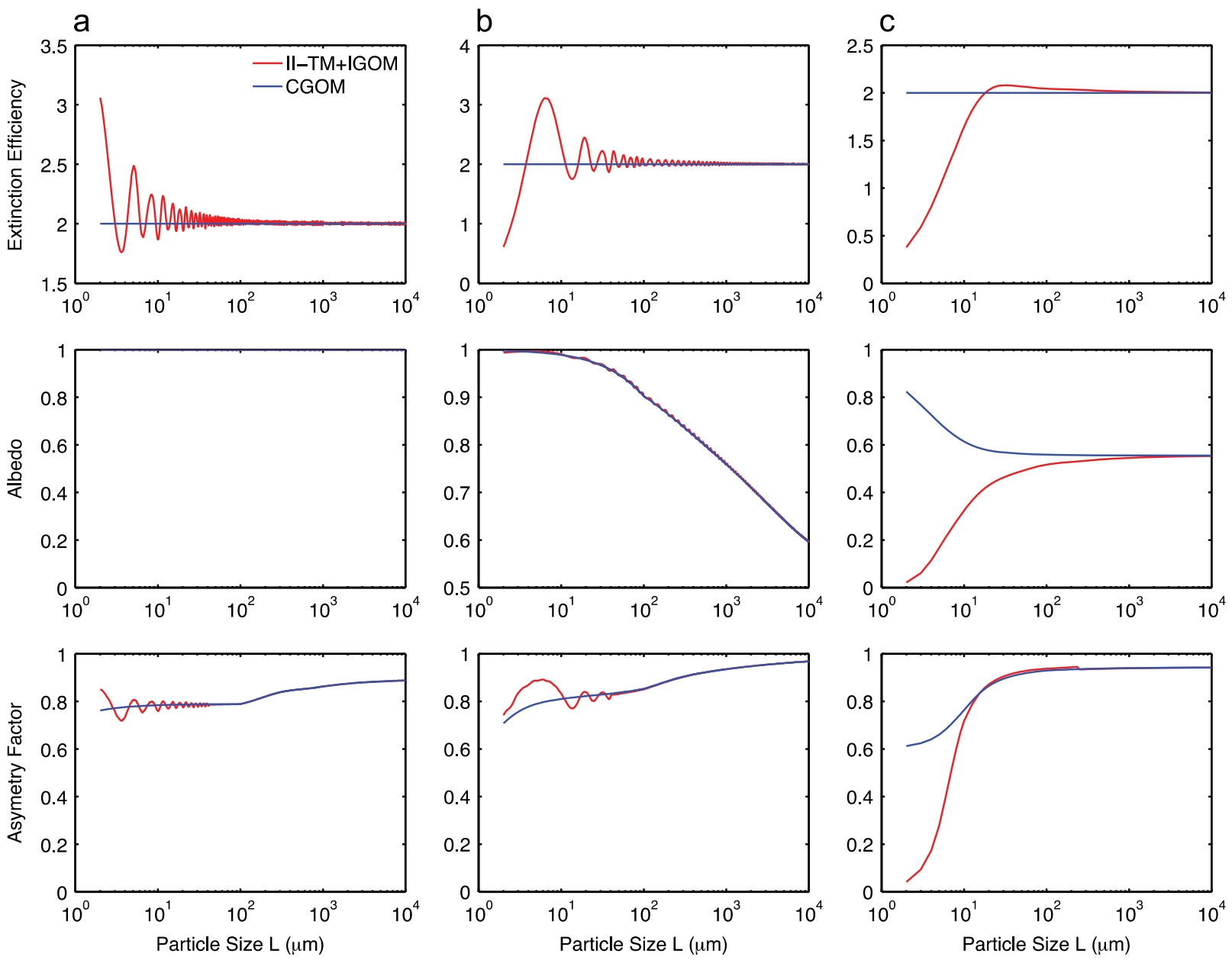

Fig. 3. Comparison of the extinction efficiency, the single-scattering albedo, and the asymmetry factor at wavelengths (a) $0.65 \mu \mathrm{m}$ (left column), (b) $2.13 \mu \mathrm{m}$ (middle column), and (c) $12 \mu \mathrm{m}$ (right column) computed from the II-TM+IGOM and the CGOM. 
Fig. 2 shows a comparison of phase functions computed from the CGOM, the II-TM and the IGOM at six representative wavelengths. The particle sizes for each phase function are indicated in the figure. The phase functions computed from the II-TM and IGOM are very similar. At wavelengths of 0.65 and $0.86 \mu \mathrm{m}$ (Figs. 2(a) and (b)), the ice particles are non-absorptive and ice halos at $22^{\circ}$ and $46^{\circ}$ are beginning to emerge for the selected size in the II-TM and IGOM calculations, but they are much too prominent in the CGOM results. However, a peak that is predicted by the IGOM and the CGOM at approximately $150^{\circ}$ is not observed in the IITM simulated phase function. The size parameter is most likely insufficiently large, so that the ray-concept in the CGOM and the IGOM fails for higher-order rays. Additionally, a small peak of phase function at the wavelength of $8.5 \mu \mathrm{m}$ predicted from the CGOM is not observed from the II-TM simulation. Because the II-TM is computationally expensive and the II-TM and the IGOM results are very close as shown in Fig. 2, we only employ the IGOM in the following simulations when the particle sizes are larger than the numbers indicated in the figure. The IGOM results of the extinction efficiency and the absorption efficiency are adjusted with respect to the II-TM for a smooth transition through a semi-empirical estimation of the edge effect contribution [46].

Fig. 3 shows a comparison of the extinction efficiency $\left(Q_{\text {ext }}\right)$, the single-scattering albedo $(\omega)$, and the asymmetry factor $(g)$ of hexagonal columns at three wavelengths $(0.65$, 2.13 , and $12 \mu \mathrm{m})$, computed from the CGOM and II$\mathrm{TM}+\mathrm{IGOM}$, and for particle sizes ranging from 2 to $10,000 \mu \mathrm{m}$. The differences between the results from the two techniques are evident, particularly for small particle sizes. Note that the extinction efficiencies and the asymmetry factors at the wavelengths of 0.65 and $2.13 \mu \mathrm{m}$ oscillate, but the CGOM results do not because the interference effect is not taken into account. While the single scattering albedo is well approximated by the CGOM at $2.13 \mu \mathrm{m}$, large errors for small particles occur at $12 \mu \mathrm{m}$. Note that the size parameter for a given particle size at $12 \mu \mathrm{m}$ is approximately 5.6 times smaller than that at $2.13 \mu \mathrm{m}$ and the particle is more absorptive at $12 \mu \mathrm{m}$ than at $2.13 \mu \mathrm{m}$. As is evident from the comparison, the T-matrix and IGOM solutions cover a size parameter range where the conventional geometric-optics principles break down.

Based on the independent scattering assumption, ice cloud bulk-scattering properties are obtained by integrating the single-scattering properties of ice particles with the particle-size distributions (PSD). The Gamma size distribution is adopted in this study, and the PSD can be represented by the following function:

$n(L)=N_{o}(L / \nu)^{\alpha-1} \exp (-L / \nu)$

where $N_{o}$ is a normalization factor and $L$ is the maximum dimension (length of the column). The PSD is determined by $\alpha$ and $\nu$, the shape and scale parameters. To determine the two parameters, we use the values from ice cloud in situ measurements [57] and from retrievals [58] for contrails. Fig. 4 shows the relationship between $\alpha$ and $\nu$. The cirrus PSD data compiled by Baum et al. [57] are illustrated by blue dots in the figure, and the retrieved data from Iwabuchi et al. [58] are shown as green circles. The

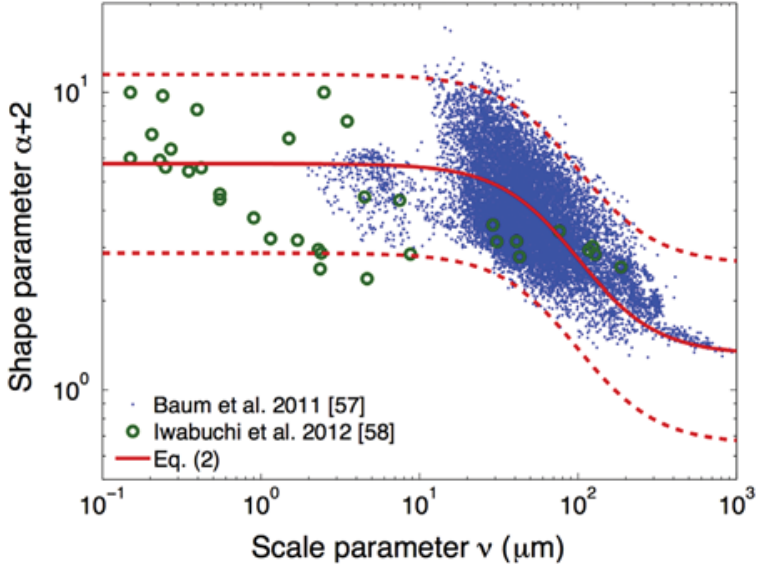

Fig. 4. Size distributions used in the computation of ice cloud bulkscattering properties. The red dashed curves in the figure indicate that most data lay in the range either twofold smaller or larger than the fitting curve. (For interpretation of the references to color in this figure legend, the reader is referred to the web version of this article.)

data show a large dispersion. Different from the linear fit given by Iwabuchi et al. [58], this study uses a hyperbolic tangent function to fit the observations, and the solid red curve in the figure follows:

$\log (\alpha+2)=0.44-0.32 \times \tanh [2 \times \log (v)-4]$.

A total of 18 pairs of $\alpha$ and $\nu$ values corresponding to the effective particle diameter $D_{\text {eff }}$ ranging from 10 to $180 \mu \mathrm{m}$ at an interval of $10 \mu \mathrm{m}$ are used in this study. $D_{\text {eff }}$ is defined by

$D_{e f f}=\frac{3\langle V\rangle}{2\langle A\rangle}$

where $\langle V\rangle$ and $\langle A\rangle$ are the averaged volume and projected area of the particles associated with a given size distribution. The dashed curves in the figure indicate that most data lay in the range either twofold smaller or larger than the fitting curve.

Fig. 5 compares the bulk-scattering phase functions computed from the CGOM and the II-TM+IGOM at 0.65 , 2.13 , and $12.0 \mu \mathrm{m}$. The differences are evident when the $D_{e f f}$ is small, but become negligible when $D_{\text {eff }}$ becomes sufficiently large. Indicated in the figure are the bulk extinction efficiency, the bulk single-scattering albedo, and the bulk asymmetry factor. The edge-effect contribution to the extinction efficiency is observable. In the case of a large $D_{\text {eff }}$, small particles contribute less to the total cross section because of their smaller projected area and relatively low weight of probability in the particle size distribution. The difference between the extinction/scattering cross-sections computed by the II-TM+IGOM and the CGOM decrease as the $D_{\text {eff }}$ increases.

From the comparison of the single-scattering properties computed from the CGOM and the "benchmark", we identify errors in optical properties of small ice crystals calculated using CGOM. In the following sections, we quantify the radiative uncertainties if the CGOM is applied to all particle size parameters. 

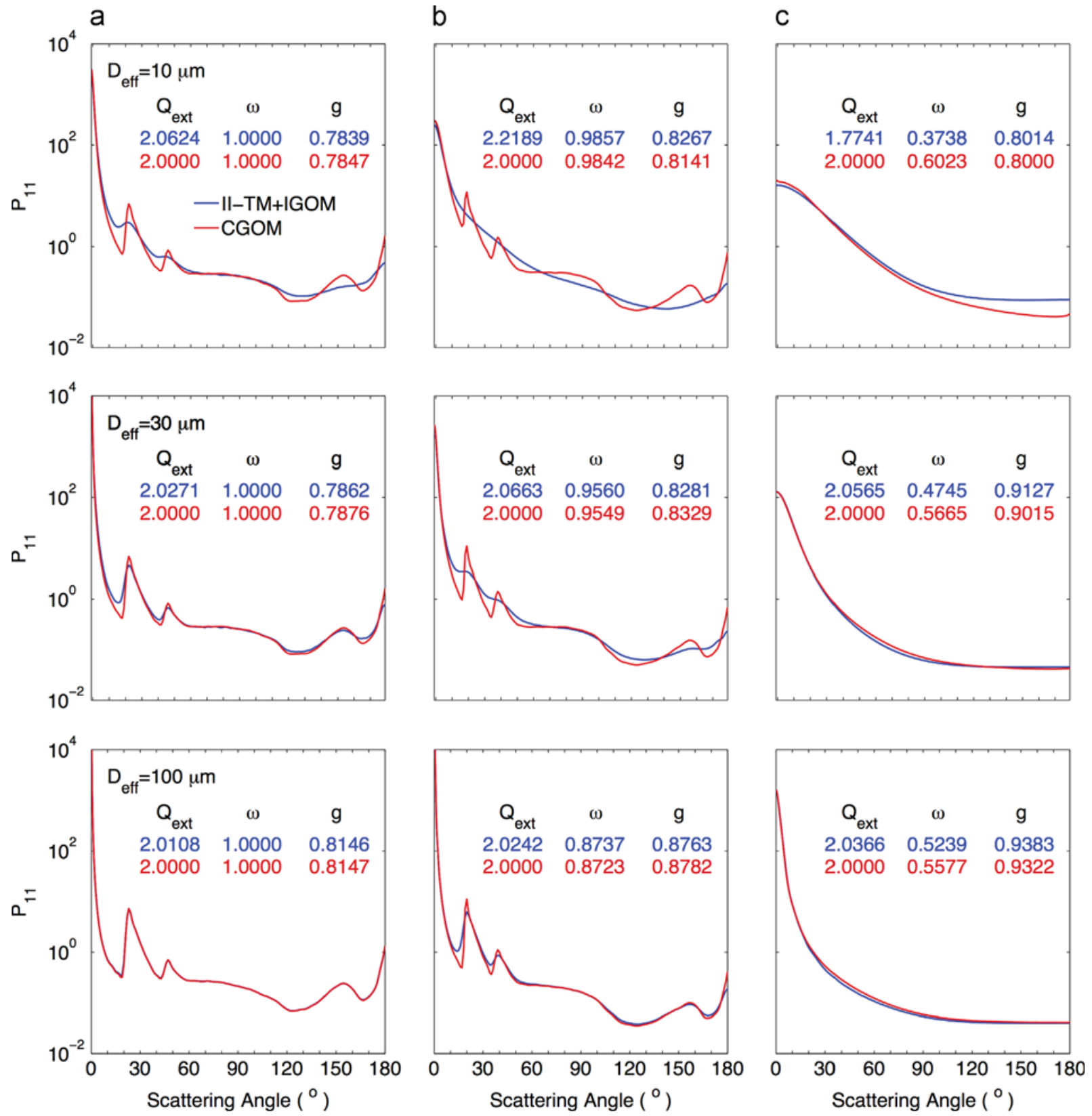

Fig. 5. Comparison of bulk ice cloud scattering phase functions $\left(P_{11}\right)$ for different effective diameters $\left(D_{\text {eff }}\right)$ at the wavelengths of (a) $0.65 \mu \mathrm{m}($ left column), (b) $2.13 \mu \mathrm{m}$ (middle column), and (c) $12.0 \mu \mathrm{m}$ (right column). Indicated in the figure are the bulk extinction efficiency ( $Q_{\text {ext }}$ ), the bulk single-scattering albedo $(\omega)$, and the asymmetry factor $(g)$ of the bulk-scattering phase function.

\section{Remote sensing implications}

The previous section compared both the single- and bulk-scattering properties of hexagonal columns computed from the II-TM+IGOM and the CGOM. The effect of the bulk-scattering property differences on the remote sensing implementations is assessed in this section. Two independent retrieval methods are used that employ the hexagonal column model and the scattering properties obtained from the II-TM+IGOM and the CGOM. One retrieval method is based on two solar reflectance bands and the other on three infrared (IR) bands. The accuracy of the CGOM is evaluated by comparing the retrieved ice cloud properties (i.e., cloud optical thickness $\tau$ and $D_{\text {eff }}$ ) with those obtained from the II-TM+IGOM simulations for these two methods.

Both retrieval methods use measurements from the Moderate Resolution Imaging Spectroradiometer (MODIS) on the National Aeronautics and Space Administration (NASA) Terra and Aqua platforms. MODIS has 36 spectral bands ranging from 0.41 to $14.2 \mu \mathrm{m}$. In the current study, we employ the Aqua/MODIS level-1B (L1B) collection-5 
("MYD021KM") product that provides top of the atmosphere (TOA) radiances/reflectivities for the 0.86- and 2.13- $\mu \mathrm{m}$ bands and the brightness temperature for three IR bands (8.5-, 11- and 12- $\mu \mathrm{m}$ ), which are used in the retrieval procedures, and a product ("MOD03") that contains the geolocation and viewing geometry information for the satellite observations. The MODIS-retrieved cloud phase, optical thickness, and top height from the Aqua/ MODIS level-2 cloud product (MYD06) are also used. The MODIS pixels that are over the ocean and identified as ice clouds in the MODIS level-2 products are used for our retrievals. For the IR retrieval, which is inherently less sensitive to optically thick clouds, the cases with MODIS optical thicknesses larger than 6 are neglected. The atmospheric profiles used in the RT calculations are provided by the Modern Era Retrospective-Analysis for Research and Applications (MERRA) model, specifically the 3-hourly profiles ("Int3_3d_ams_CP") of temperature, water vapor density, and ozone density defined for 42 pressure levels at a grid resolution of $1.25^{\circ} \times 1.25^{\circ}$.

The first retrieval algorithm is based on two solar reflectance bands: specifically, a weakly absorbing, visible or near-infrared window band (VIS/NIR) (e.g., 0.64 or $0.86 \mu \mathrm{m}$ ) that is sensitive mainly to $\tau$, and an ice absorbing shortwave infrared (SWIR) band (e.g., 1.6 or $2.13 \mu \mathrm{m}$ ) that is sensitive to both $\tau$ and $D_{\text {eff }}$ [59]. This study uses the MODIS observations at 0.86 and $2.13 \mu \mathrm{m}$ bands, and the fast radiative transfer model (RTM) developed by Wang et al. [60] to simulate the reflectance of the modeled ice clouds. The fast RTM uses pre-computed bidirectional reflectance/transmittance distribution functions (BRDF/ BTDF) for single layer clouds, and lookup tables (LUTs) for different $\tau$ and $D_{\text {eff }}$ are calculated using the discrete ordinates radiative transfer program (DISORT). Fig. 6 shows the comparisons of the LUTs built on the optical properties calculated by the II-TM+IGOM and the CGOM, respectively. The solar zenith angle is assumed to be $20^{\circ}$. Two viewing zenith angles, $37^{\circ}$ and $70^{\circ}$, are selected for radiative transfer simulations to build LUTs as shown in Fig. 6a and b, respectively. Substantial differences exist in the $D_{\text {eff }}$ isolines, because the differences between the optical properties given by the II-TM+IGOM and the CGOM become larger as $D_{\text {eff }}$ decreases. Furthermore, as shown in Fig. 6, the relative differences between the LUTs are sensitive to the solar-satellite geometry, because the relative magnitude between the CGOM and the IITM+IGOM phase functions change with respect to the scattering angles (see Fig. 5). For the case in Fig. 6a, the $D_{\text {eff }}$ isolines computed from the CGOM optical properties are lower than the II-TM+IGOM counterparts, which implies that the retrieved $D_{\text {eff }}$ based on the CGOM in all probability be smaller than those based on the II-TM+IGOM. However, for the case in Fig. 6b, the CGOM optical properties result in higher reflectivities at the same $D_{\text {eff, suggesting }}$ that the retrieved $D_{\text {eff }}$ is mostly likely to be overestimated. The two LUTs of the CGOM and the II-TM+IGOM in Fig. 6a and b converge as $D_{\text {eff }}$ reaches $100 \mu \mathrm{m}$ and $30 \mu \mathrm{m}$, respectively. In terms of the $\tau$ isolines shown in Fig. 6, the results for the II-TM+IGOM and CGOM are similar with noticeable differences for small $D_{\text {eff. }}$.

To retrieve the cloud optical thickness and effective particle diameter, the following cost function is defined

$\chi^{2}=\left(\frac{R_{o, 0.86}-R_{s, 0.86}}{R_{o, 0.86}}\right)^{2}+\left(\frac{R_{o, 2.13}-R_{s, 2.13}}{R_{o, 2.13}}\right)^{2}$,

where $R_{o, \lambda}$ and $R_{s, \lambda}$ are the MODIS bidirectional reflectivities and the fast RTM simulations at wavelength $\lambda$, respectively. The $\tau$ and $D_{\text {eff }}$ values are obtained from the simulated reflectivities that minimize Eq. (4). Fig. 7 illustrates the cloud properties retrieved from the MODIS measurements. A MODIS granule at 07:45 UTC on August 2, 2010 is used in the retrieval. Fig. $7 a$ and $b$ shows the solar zenith and viewing zenith angles, respectively. Fig. 7c and d provides the retrieved cloud $\tau$ and $D_{\text {eff }}$ based on the LUT developed from the scattering properties given by the II-TM+IGOM. As illustrated from the retrieved results, the clouds in this granule have a wide range of $\tau$ and $D_{\text {eff }}$ values.

The relative differences between the II-TM+IGOM and CGOM cases are shown in Fig. 7e and f, and the relative differences are given by

$R E_{\tau}=\frac{\tau_{C G O M}-\tau_{I I-T M+I G O M}}{\tau_{I I-T M+I G O M}} \times 100 \%$
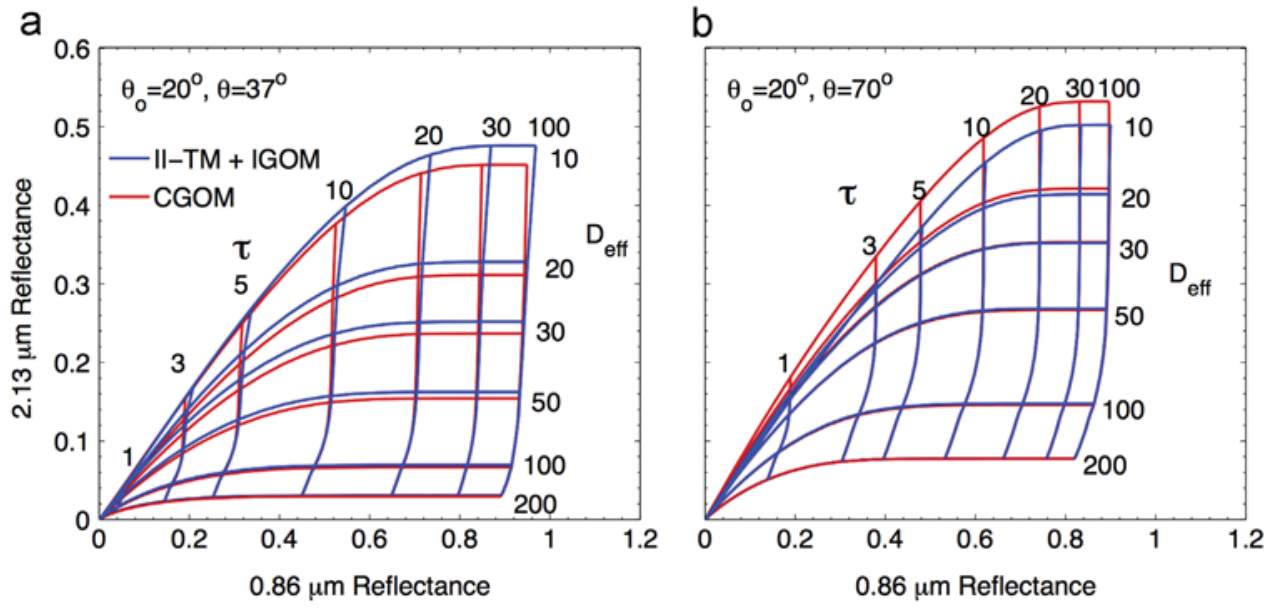

Fig. 6. Comparisons of the LUTs using 0.86 - and $2.13-\mu \mathrm{m}$ reflectance calculated using scattering properties from the II-TM+IGOM and the CGOM. 
a

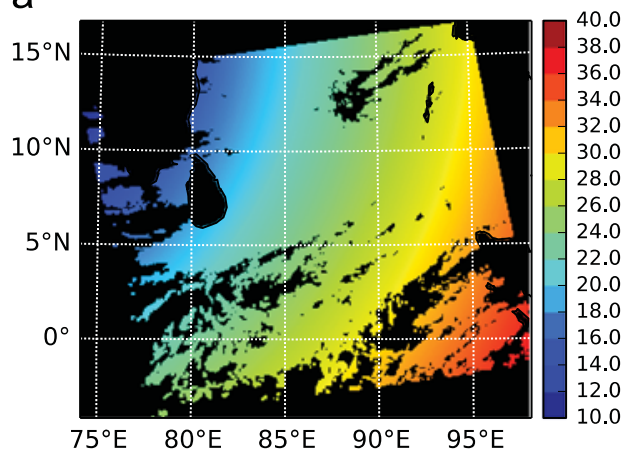

C

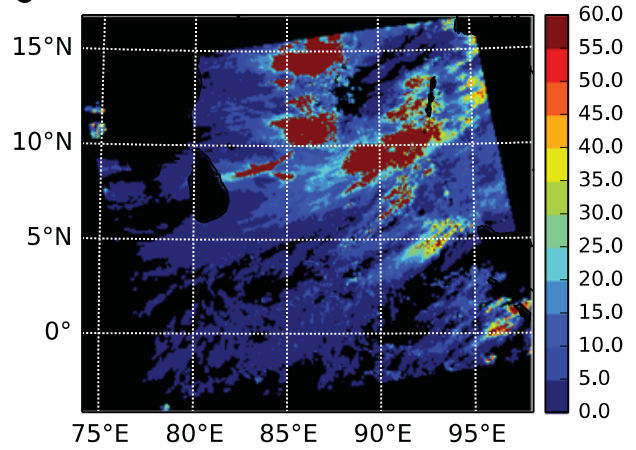

e

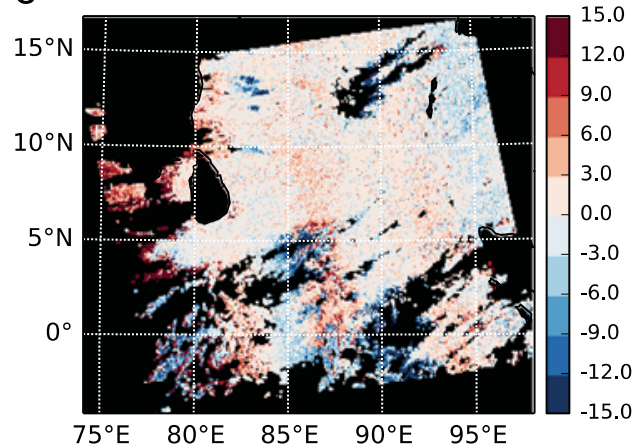

$\mathrm{b}$

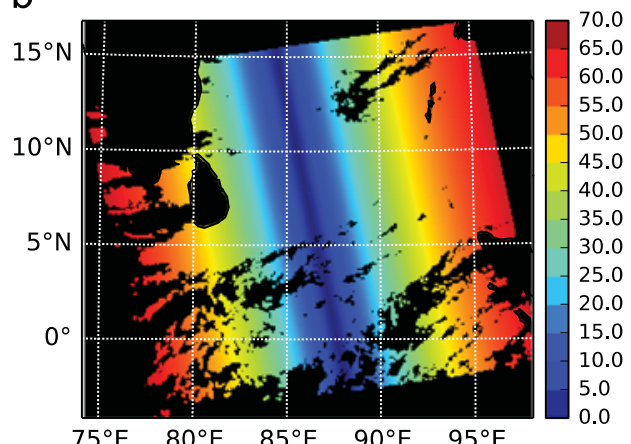

d

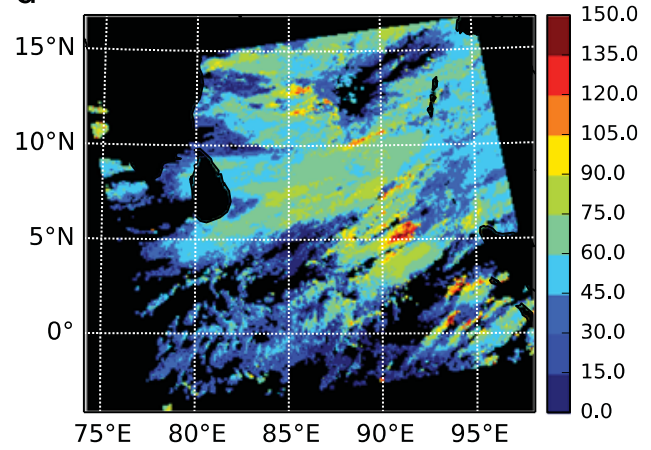

f

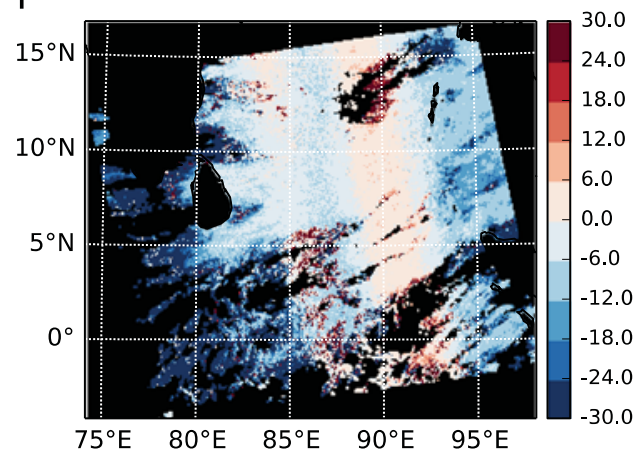

Fig. 7. The solar zenith (a) and view zenith (b) for the MODIS observations, the retrieved cloud optical thickness (c), and the retrieved effective diameter (d) based on the II-IM + IGOM, and the relative differences of the retrieved optical thickness (e) and effective diameter (f) based on the II-TM+IGOM and the CGOM databases.

and

$R E_{D_{e f f}}=\frac{D_{e f f, C G O M}-D_{e f f, I I-T M+I G O M}}{D_{e f f, I I-T M+I G O M}} \times 100 \%$

where $\tau_{I I-T M+I G O M}$ and $D_{\text {eff,II-TM+IGOM}}$, and $\tau_{C G O M}$ and $D_{\text {eff.CGOM }}$ are the values retrieved with the scattering properties given by the II-TM+IGOM and the CGOM, respectively. As shown in Fig. $7 \mathrm{e}$ and $\mathrm{f}$, the relative differences caused by the CGOM are as large as $15 \%$ and

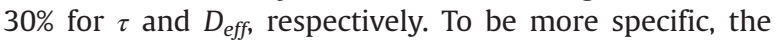
relative differences of $\tau$, generally under $10 \%$, show weak dependence on the satellite viewing geometry, and the largest differences occur at those pixels with small $\tau$. However, it should be noted that the relative differences on the retrieved $D_{\text {eff }}$ shown in Fig. $7 \mathrm{f}$ are much larger, and strongly depend on the viewing zenith angle. The correlation between Fig. $7 \mathrm{~b}$ and $\mathrm{f}$ can be explained by the LUTs shown in Fig. 6. The CGOM-based $D_{\text {eff }}$ isolines in the LUTs can be lower or higher than the II-TM+IGOM counterparts because the reflectivity for a specific solarsatellite configuration is highly related to the magnitude of the phase functions at the corresponding scattering angles where the CGOM phase function can be larger or smaller than the II-TM+IGOM phase function. Consequently, the differences of retrieved effective diameters from the two LUTs can be smaller or larger with a similar pattern as the viewing zenith angle.

Fig. 8 shows the mean biases (solid line) and the standard deviations (shaded areas) of the retrieved $\tau$ and $D_{\text {eff }}$ values for the CGOM-derived scattering properties. It can be seen that the retrieved $\tau$ is quite accurate for $\tau \leq 30$ with mean biases of less than $1 \%$ and standard deviations 

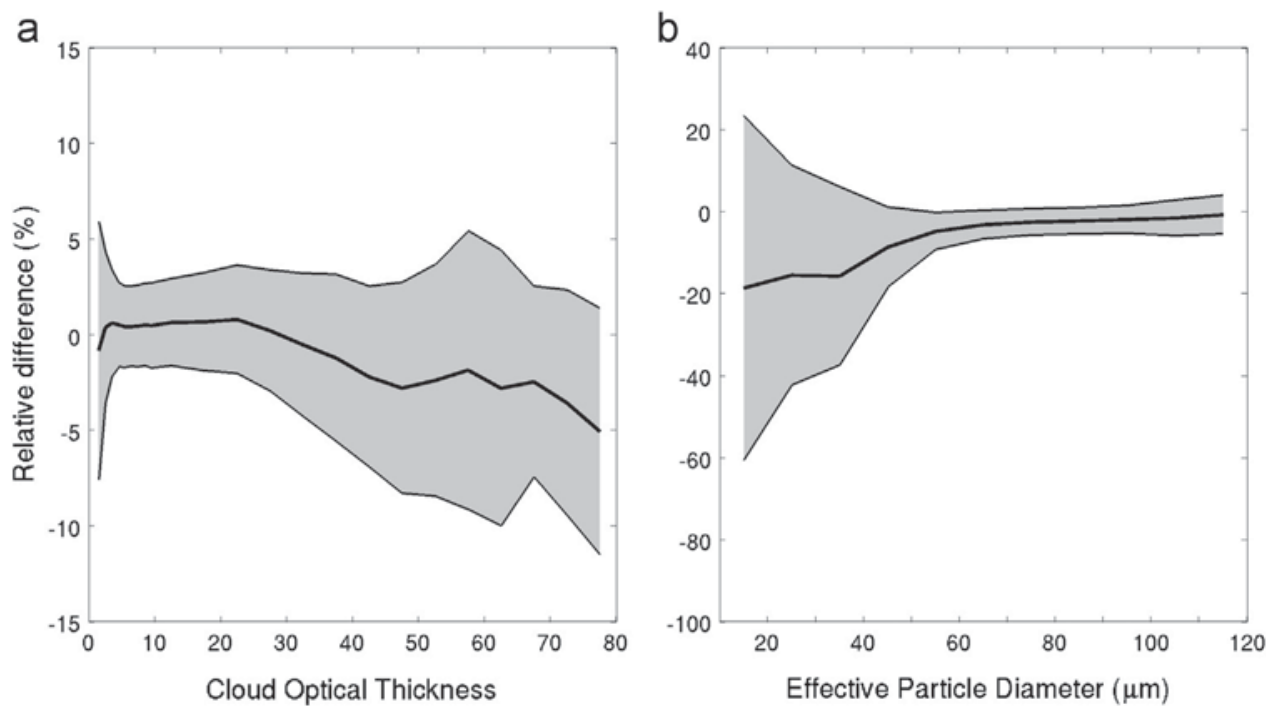

Fig. 8. The mean and standard deviations for the relative difference in the retrieved optical thickness (a) and effective diameter (b).

less than $5 \%$, but the CGOM-based retrievals underestimate $\tau$ by approximately $5 \%$ and have standard deviations that can exceed $5 \%$ as $\tau$ increases. Note that, the reflectance at $0.86 \mu \mathrm{m}$ becomes less sensitive to $\tau$ for thicker clouds. For example, the reflectivity increases by an amount $<0.1$ as the $\tau$ increases from 30 to 100 for all $D_{\text {eff. }}$. Low sensitivity indicates the existence of large retrieval differences for thick clouds. Meanwhile, the relative differences for the retrieved $D_{\text {eff }}$ are more significant with mean biases and standard deviations of approximately up to $20 \%$ and $40 \%$, respectively, for the particles with $D_{\text {eff }} \leq 50 \mu \mathrm{m}$. However, the errors become much smaller as $D_{\text {eff }}$ increases.

Fig. 9 shows the relative differences in CGOM-retrieved $D_{\text {eff }}$ within different ranges of $\tau$. As evident from the figure, the errors of $D_{\text {eff }}$ are very sensitive to $\tau$. For example, the patterns in Fig. 9a and b are quite different and very large bias are obtained for optically thin clouds with small particle sizes. Note that the pixels for $D_{\text {eff }}>\sim 85$ are not available when $\tau<1$. In comparison of Fig. 9c and d, the errors of $D_{\text {eff }}$ are relatively larger for $\tau<2$ than those for $\tau>2$. As shown in Fig. 9e and f, the errors in $D_{\text {eff }}$ are most significant for cases where $\tau<4$, and much smaller as $\tau>4$. Because Fig. 9e is similar to Fig. 8b, the large errors shown in Fig. 8b primarily occur when $\tau<4$. Different from the above findings, the retrieved $\tau$ is found not to be very sensitive to $D_{\text {eff. }}$. For example, the pattern of the relative differences of $\tau$ are very similar for the pixels with $D_{\text {eff }}<50 \mu \mathrm{m}$ as for those with $D_{\text {eff }} \geq 50 \mu \mathrm{m}$ (figure not shown).

The second retrieval method is based on the three MODIS IR bands at $8.5,11$, and $12 \mu \mathrm{m}$. The fast high-spectralresolution RTM (HRTM) developed by Wang et al. [61,62] is used to simulate the radiances and resulting brightness temperatures for the three bands. Gaseous absorption is taken into account by a pre-computed transmittance database using a rigorous line-by-line radiative transfer model (LBLRTM), and the scattering properties given by the II$\mathrm{TM}+\mathrm{IGOM}$ or the CGOM are used to calculate the angular- dependent ice cloud reflectance and transmittance, the effective emissivity, and the effective temperature functions. The spectral response functions of the three MODIS bands are considered in the HRTM. The $\tau$ - $D_{\text {eff }}$ pair that leads to the least root mean square (RMS) value is chosen as the most appropriate inference of the ice cloud properties, and the RMS is defined as

$R M S=\left[\frac{\left(B T_{o, 8.5}-B T_{S, 8.5}\right)^{2}+\left(B T_{o, 11}-B T_{S, 11}\right)^{2}+\left(B T_{o, 12}-B T_{S, 12}\right)^{2}}{3}\right]^{1 / 2}$,

where $B T_{o, \lambda}$ and $B T_{s, \lambda}$ are the brightness temperatures from MODIS observations and the HRTM simulations at wavelength $\lambda$. Fig. 10 is the same as Fig. 8 but for $\tau$ and $D_{\text {eff }}$ from the IR retrieval. The retrievals carried out using the scattering properties from the CGOM systemically overestimate the $\tau$ by approximately $12 \%$ with standard deviations over $3 \%$. For $D_{\text {eff, }}$ the mean biases caused by the inaccuracy of the CGOM range between about $-10 \%$ and $10 \%$ with standard deviations up to $20 \%$.

\section{Uncertainties in radiative forcing calculations}

The differences in the ice cloud optical properties between the II-TM + IGOM and CGOM cases also influence the parameterization schemes of ice cloud bulk optical properties used in the single-column radiative transfer models (RTMs), as well as in general circulation models (GCMs), thereby affecting the simulations of ice cloud broadband radiances. Results are shown for bands in the shortwave (SW) spectrum $(\lambda<5 \mu \mathrm{m})$ and in the longwave (LW) spectrum $(5<\lambda<100 \mu \mathrm{m})$. The cloud radiative effect (CRE) is defined as the difference in the net TOA/surface flux (SW, LW or total) between the cloudy sky and the clear sky. 

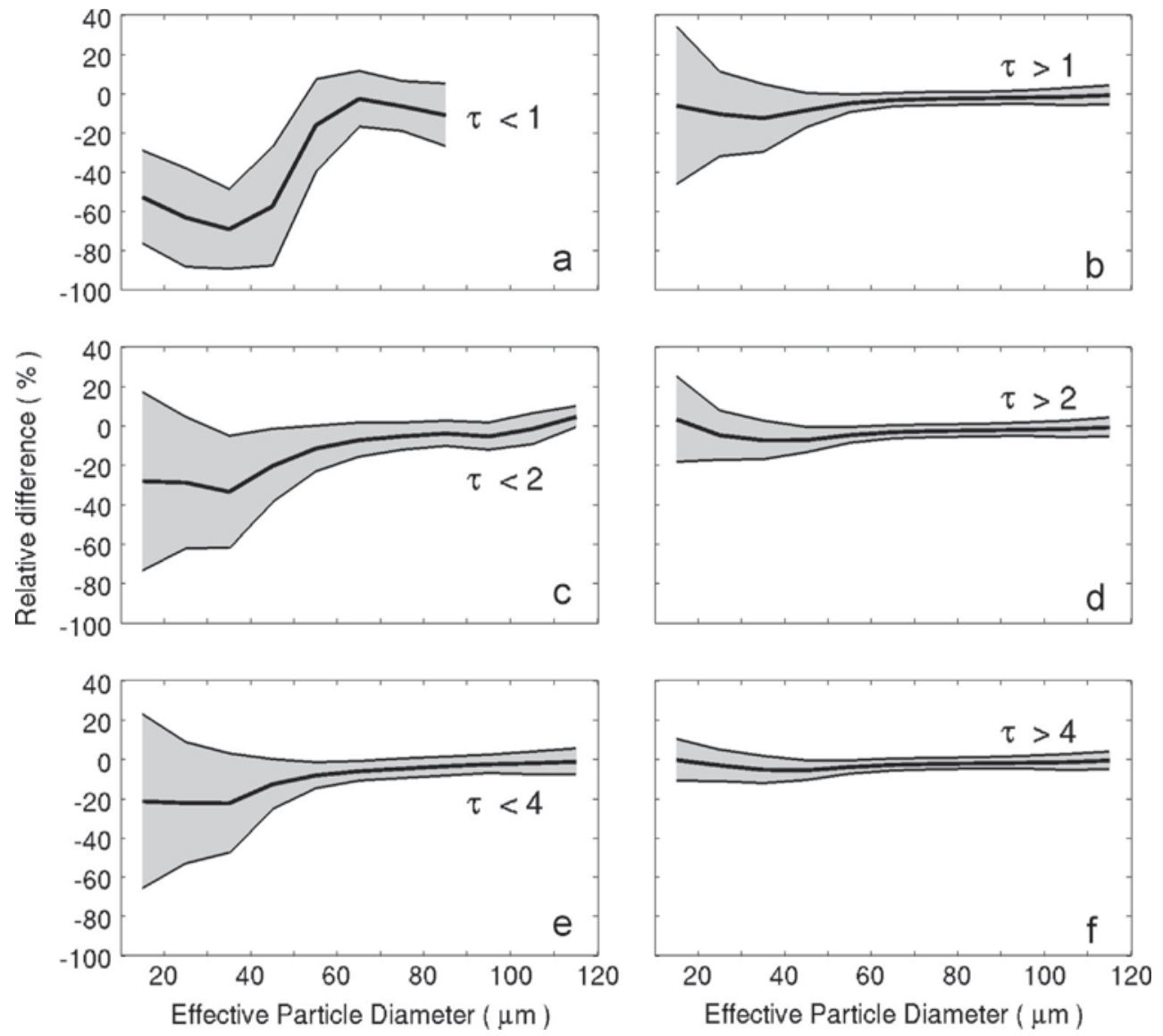

Fig. 9. The mean and standard deviations for the relative difference in the retrieved effective diameter within different ranges of the optical thickness.
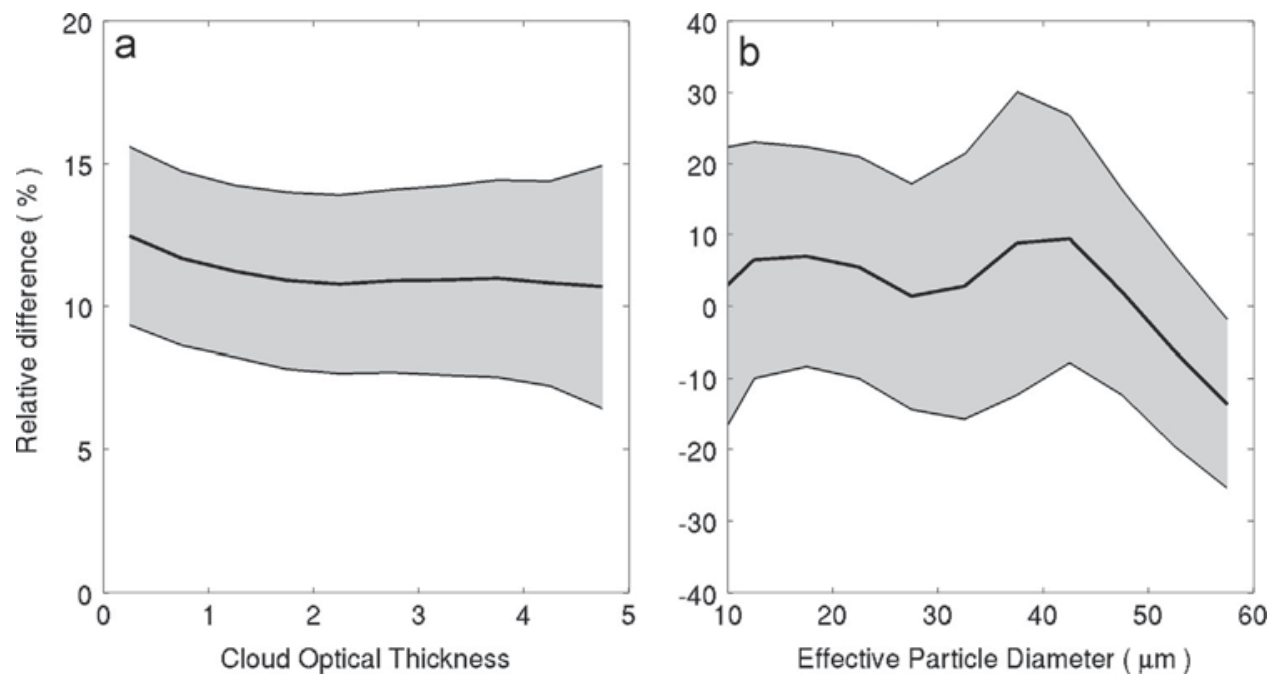

Fig. 10. The mean and standard deviations for the relative difference in the retrieved optical thickness (a) and effective diameter (b) for the IR retrieval.

The optical properties of ice clouds used in numerical model simulations include the mass extinction coefficient $\left(k_{\text {ext }}\right)$, the mass absorption coefficient $k_{a b s}$, the bulk singlescattering albedo $\bar{\omega}$, and the mean asymmetry factor $\bar{g}$, which are defined by

$$
k_{\text {ext }}=\frac{\int_{\lambda_{\min }}^{\lambda_{\max }} \int_{L_{\min }}^{L_{\max }} Q_{\text {ext }}(L, \lambda) A(L) S(\lambda) n(L) d L d \lambda}{\int_{\lambda_{\min }}^{\lambda_{\max }} \int_{L_{\min }}^{L_{\max }} V(L) S(\lambda) n(L) d L d \lambda},
$$


a

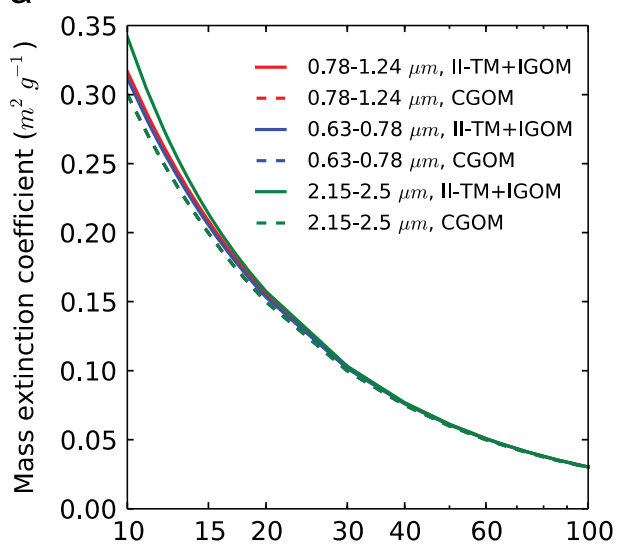

C

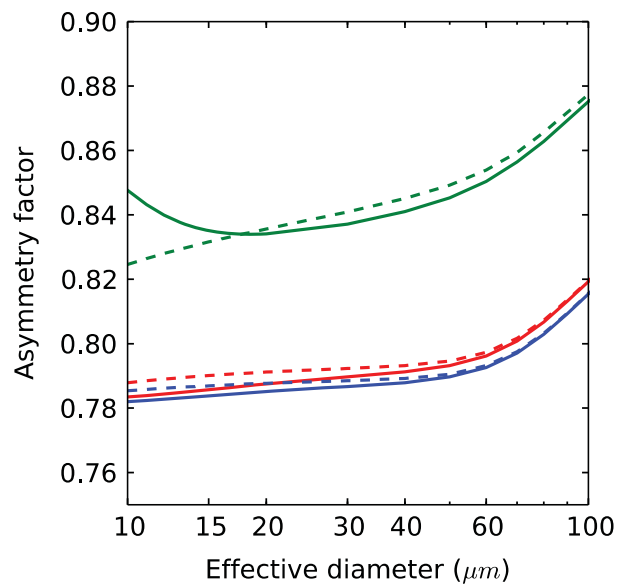

b

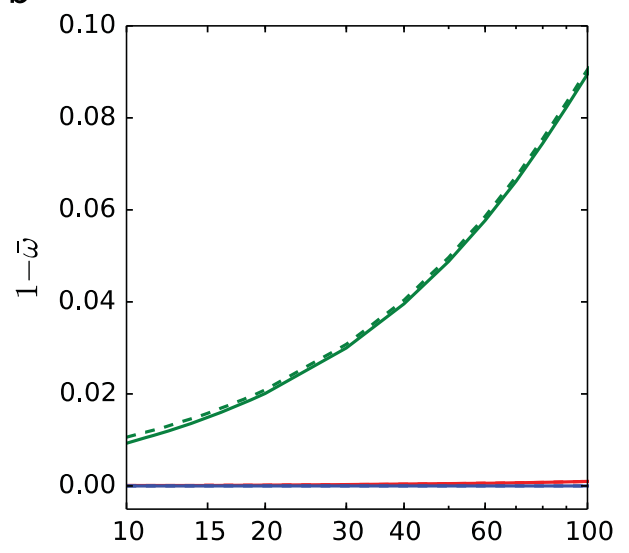

d

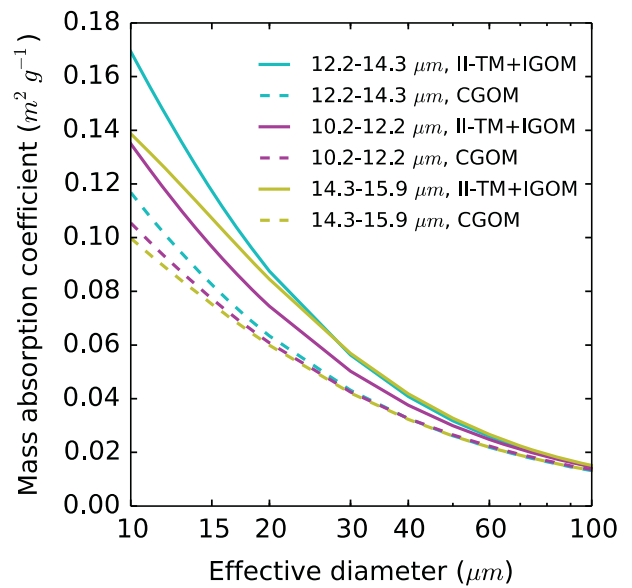

Fig. 11. Comparison of bulk ice cloud optical properties derived from II-TM+IGOM and CGOM at selected spectral bands of the RRTMG RTM.

$k_{a b s}=\frac{\int_{\lambda_{\min }}^{\lambda_{\max }} \int_{L_{\min }}^{L_{\max }} Q_{a b s}(L, \lambda) A(L) S(\lambda) n(L) d L d \lambda}{\int_{\lambda_{\min }}^{\lambda_{\max }} \int_{L_{\min }}^{L_{\max }} V(L) S(\lambda) n(L) d L d \lambda}$,

$\bar{\omega}=1-k_{a b s} / k_{e x t}$,

$\bar{g}=\frac{\int_{\lambda_{\min }}^{\lambda_{\max }} \int_{L_{\min }}^{L_{\max }} g(L, \lambda) Q_{s c a}(L, \lambda) A(L) S(\lambda) n(L) d L d \lambda}{\int_{\lambda_{\min }}^{\lambda_{\max }} \int_{L_{\min }}^{L_{\max }} Q_{s c a}(L, \lambda) A(L) S(\lambda) n(L) d L d \lambda}$,

where $L$ is the height of the hexagonal column in a particle size range $\left[L_{\min }, L_{\max }\right], V$ and $A$ are the volume and the average projected area, $\lambda_{\min }$ and $\lambda_{\max }$ specify the wavelength limits of a given SW/LW band, and $S(\lambda)$ is the solar spectrum for SW bands and the Planck function (the cloud temperature is assumed to be $233 \mathrm{~K}$ ) for LW bands. All are parameterized as functions of ice cloud $D_{\text {eff. }}$. Fig. 11 shows the ice cloud bulkscattering properties for selected spectral bands of the RRTMG radiative transfer model. For the CGOM case, the mass extinction coefficient is invariant with the SW spectral bands, and will cause a difference in the mass extinction coefficient of up to $0.05 \mathrm{~m}^{2} \mathrm{~g}^{-1}$ (14\%) for the $2.15-2.5 \mu \mathrm{m}$ band for small $D_{\text {eff. }}$ Similarly, the mass absorption coefficient for a given LW band (e.g., the $14.3-15.9 \mu \mathrm{m}$ band) can differ by about $0.04 \mathrm{~m}^{2} \mathrm{~g}^{-1}(24 \%)$. The largest differences in the asymmetry factor occur for small $D_{\text {eff }}$ in the longer wavelength bands. Relatively small differences are found for the single-scattering albedo (SSA), where the II-TM+IGOM case has slightly lower SSA than the CGOM case for the near IR band.

Although differences in optical properties are evident, it is useful to understand how these differences affect the simulated ice cloud CRE, especially for single bands in the SW and LW spectrum. Here, the RRTMG SW/LW model [64] is implemented with a standard mid-latitude summer atmospheric profile, assuming a solar zenith angle of $60^{\circ}$, a single-layer ice cloud located at $12 \mathrm{~km}$, a surface albedo of 0 , and an emissivity of 1 for all wavelengths. The left panel in Fig. 12 shows the differences of TOA SW, LW, and total $(\mathrm{SW}+\mathrm{LW})$ ice CRE obtained from CGOM and II-TM + IGOM databases as functions of $D_{\text {eff }}$ and $\tau$ due to the difference in optical properties. The difference is obtained by subtracting the CGOM-based CRE from the II-TM+IGOM-based CRE. The SW radiative effect is negative, meaning that II-TM+IGOM case results in a stronger ice cloud SW radiative effect than the CGOM case. The largest differences can be up to $7 \mathrm{~W} \mathrm{~m}^{-2}$ for $\tau$ ranging from 5 to 10 and $D_{\text {eff }}=10 \mu \mathrm{m}$ (i.e., small values). The LW radiative effect difference is positive, and twice as large as that of the SW at small $D_{\text {eff }}$ when $\tau$ is near 1 . The resulting total CRE 

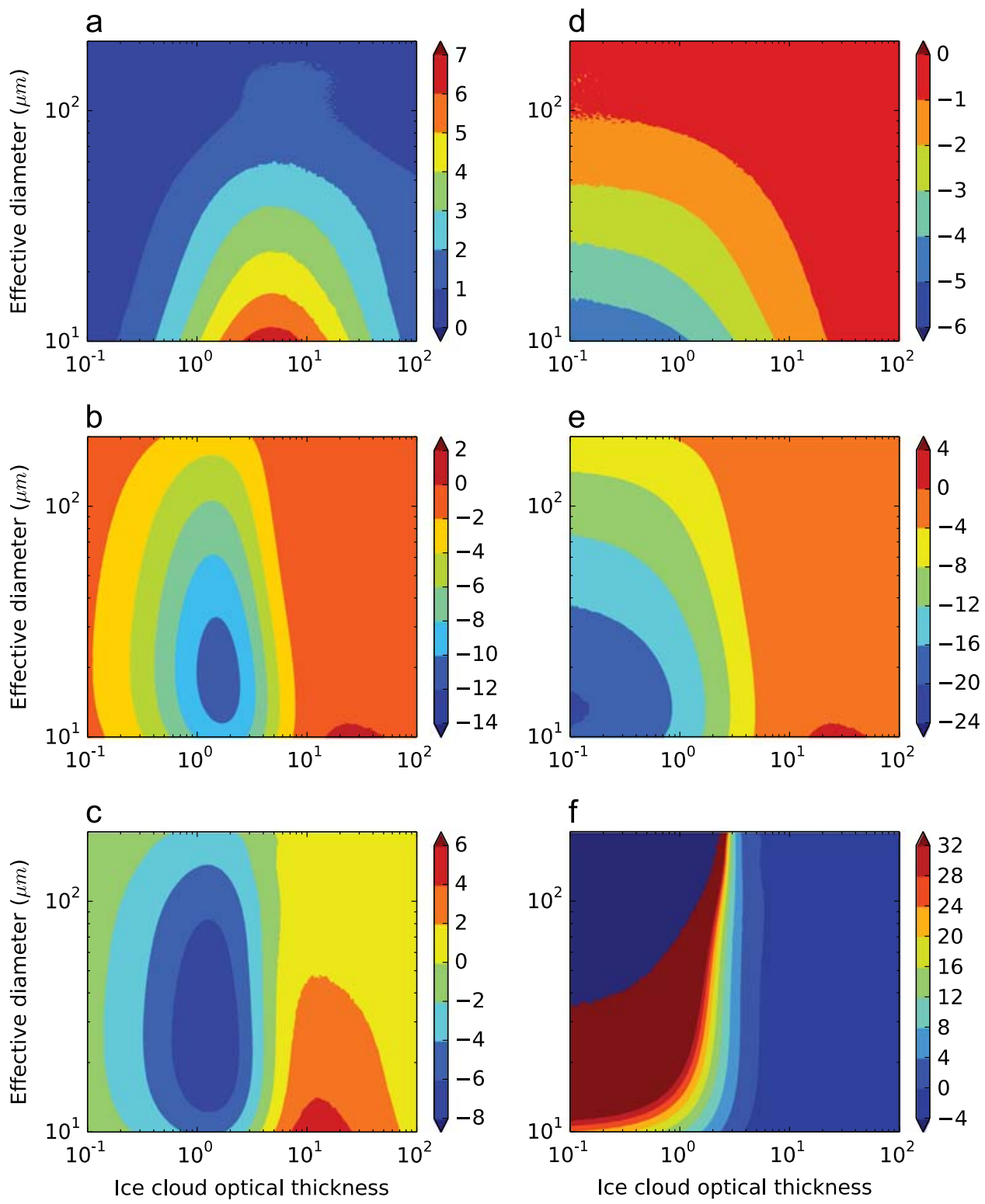

Fig. 12. Left panel: RRTMG RTM simulated TOA cloud radiative effect difference between the II-TM+IGOM and CGOM cases: solar band CRE (a), IR band CRE (b), and total (solar+IR) CRE (c). Right panel: the percentage of the relative difference in comparison with the II-TM+IGOM reference.

differences are dominated by the LW feature, resulting in positive differences for small optical thickness values $(\tau<5)$ but negative differences for large values of optical thickness $(\tau>5)$. The right panel shows relevant difference percentages. Although the differences of TOA SW tend to be small in the cases of low $\tau$ and $D_{\text {eff }}$ values, the relevant percentage differences may be significant. A similar feature is observed in the LW case.

To identify the contribution of CRE from various spectral bands of RRTMG RTM, the band-by-band CRE is calculated. Fig. 13 shows four SW/LW spectral bands with the largest contributions to the CRE difference between the II-TM+IGOM and the CGOM case. The corresponding differences in the optical properties are clearly shown in the CRE at the spectral bands.
Following Yi et al. [65], the bulk ice cloud optical properties derived from the II-TM+IGOM and the CGOM cases are implemented as parameterizations into the NCAR Community Atmospheric Model (CAM, version 5.1). A tenyear climatology is derived from the model runs with the two parameterizations, and the global annual averaged CRE are calculated and shown in Fig. 14. The SWCRE (Fig. 14a) is negative; whereas, the SWCRE difference (Fig. 14d) is generally positive, indicating that the II-TM+IGOM case results in a larger SWCRE. At the same time, as shown in Fig. 14b and e, the II-TM+IGOM case also has a stronger LWCRE. While the SW/LW CRE difference can be up to $8 \mathrm{~W} \mathrm{~m}^{-2}$ regionally, for example, in the tropical area, the global averaged SW and LW CRE differences are $0.44 \mathrm{~W} \mathrm{~m}^{-2}$ and $-1.35 \mathrm{~W} \mathrm{~m}^{-2}$, respectively. 
a

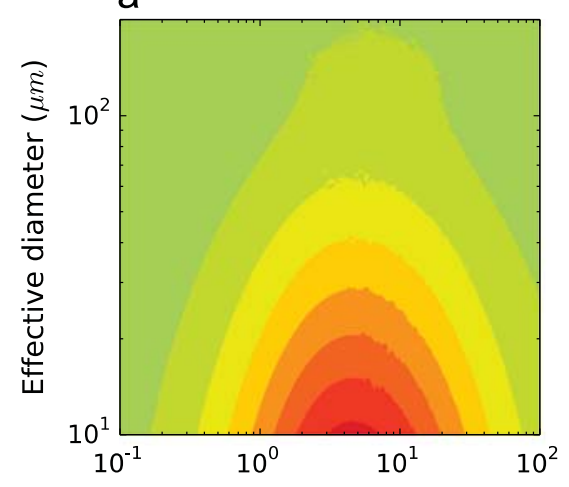

C

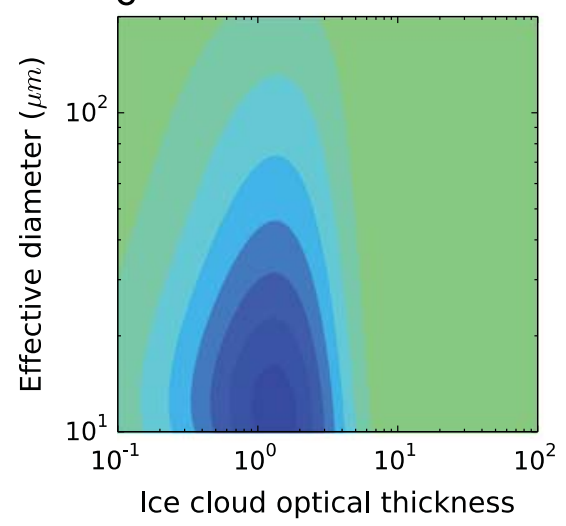

b

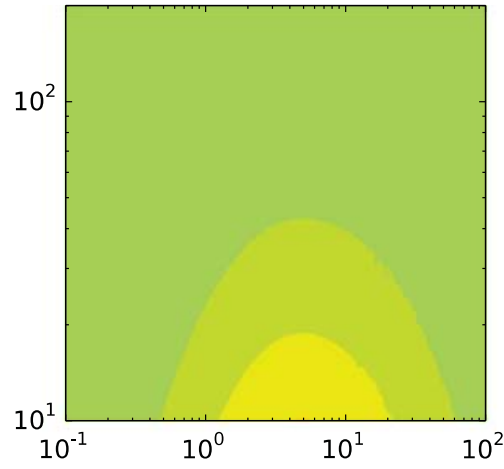

d

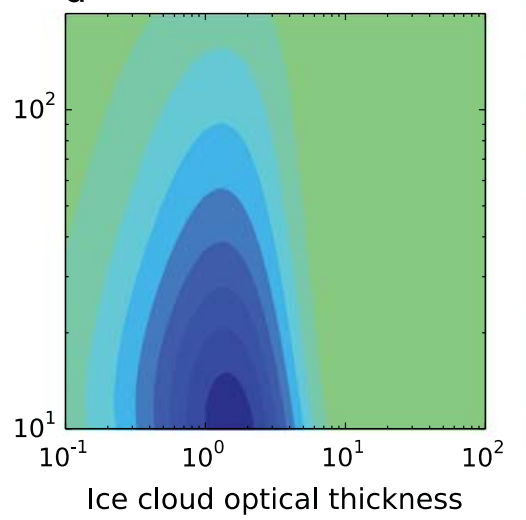

3.2

2.4

1.6

0.8

0.0

$-0.8$

$-1.6$

$-2.4$

Fig. 13. Band-by-band CRE differences between the II-TM+IGOM and the CGOM cases simulated by the RRTMG RTM at the top of the atmosphere. (a) TOA CRE $\left(W m^{-2}\right), 0.78-1.21 \mu m$, (b) TOA CRE $\left(W m^{-2}\right), 0.63-0.78 \mu m$, (c) TOA CRE $\left(W m^{-2}\right), 12.2-14.3 \mu m$ and (d) TOA CRE $\left(W m^{-2}\right), 10.2-12.2 \mu m$.

The II-TM+IGOM-based net CRE (NTCRE) and the differences between the CGOM and the II-TM+IGOM cases are shown in Fig. 14c and f, respectively.

Based on the simulated results, the biases caused by the CGOM in the LW are significantly larger than in the SW. The physical reason is that for the longwave spectrum, the particle size parameters for a relatively large range of particle sizes are small so that the accuracy of the CGOM is rather poor.

\section{Summary and conclusions}

The numerical techniques based on geometric-optics principles and its modifications provide approximate solutions for light scattering by ice crystals. In reality, the geometric-optics approximation is widely used but generally without an awareness of its uncertainties. Here, following [51], the geometric-optics methods are classified into the conventional geometric optics method (CGOM) and the improved geometric optics method (IGOM). A combination of the IGOM and the invariant imbedding T-matrix method (II-TM) is employed to derive the "benchmark" single-scattering properties of hexagonal particles with diameters from 2 to $10,000 \mu \mathrm{m}$ over a spectral range from 0.2 to $100 \mu \mathrm{m}$. Based on the single-scattering properties, we compute the ice cloud bulk-scattering properties by using the particle size distributions obtained from in situ measurements. We begin with an assessment of the uncertainties of the CGOM in the computations of single- and bulk-scattering properties. Significant biases are identified in the optical properties of small individual ice particles calculated by the CGOM. Ice cloud bulkscattering properties are applied in MODIS satellite retrieval and ice cloud forcing simulations. In the MODIS satellite retrievals using the visible and shortwave infrared, mean biases in optical thickness caused by applying the CGOM are found to be up to $5 \%$ with standard deviations of about 5\%. Mean biases and standard deviations for the retrieved effective particle diameters can be over $20 \%$ and $30 \%$, respectively, and generally decrease with particle size. However, mean biases in effective diameters for clouds with optical thicknesses above 4 are found to be below about 5\%. For retrievals using IR bands, use of CGOM leads to systematic overestimation of the optical thickness by approximately $12 \%$ and biases in effective particle diameter ranging from $-10 \%$ to $10 \%$. In the ice cloud radiative forcing calculations, biases are also found, in particular, from longwave radiative effect where the difference can be as high as $14 \mathrm{~W} \mathrm{~m}^{-2}$, although global averaged SW and LW CRE differences are $0.44 \mathrm{~W} \mathrm{~m}^{-2}$ and $-1.35 \mathrm{~W} \mathrm{~m}^{-2}$, respectively.

The sources of uncertainties in the retrieval and climate forcing calculation are primarily two-fold: the microphysical model and the accuracy of the computational 

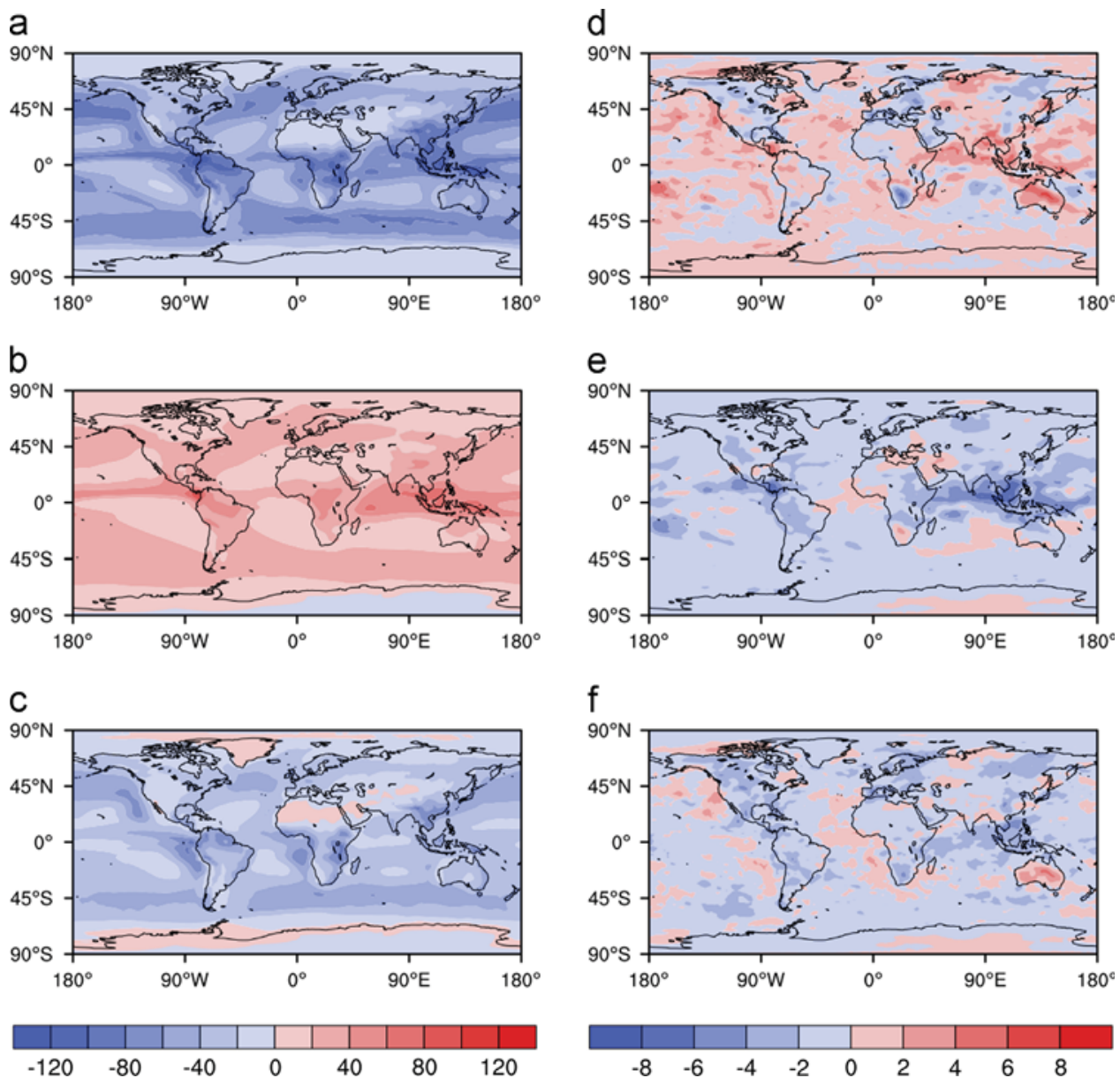

Fig. 14. Left panel: the II-TM+IGOM-based ten year mean annual CRE (unit: W m-2): SWCRE (a), LWCRE (b), and NTCRE (c). Right panel: the differences between the II-TM+IGOM and the CGOM cases (the CGOM case minus the II-TM+IGOM case).

techniques. In the present study, we only focus on the latter by assuming a simplified ice habit (i.e., smooth hexagonal column). In recent studies [63,66-68], the relative differences in retrieved optical thickness and effective particle diameter for different ice models can be comparable or larger than the maximum values found here. Similarly, in CRE calculations, differences in shortwave fluxes resulting from different microphysical models can be on the order of $10-20 \mathrm{~W} / \mathrm{m}^{2}[53,65]$ for ice particle roughness, and -70 to $-30[53]$ and -100 to $-30 \mathrm{~W} / \mathrm{m}^{2}$ $[53,69]$ for non-unity particle aspect ratios. Actually, the uncertainties from the aforementioned two sources are not independent. For example, in the analysis of model uncertainties, the techniques used in the calculation of the single-scattering properties are assumed to be accurate. Further analysis of the uncertainties will be performed in future studies.

\section{Acknowledgments}

This study was supported by a National Science Foundation (NSF) Grant (AGS-1338440), NASA Grants (NNX11AK37G and NNX11AF40G), and a subcontract (301K630) to Texas
A\&M University from the University of Wisconsin-Madison. A major portion of the simulations was carried out at the Texas A\&M University Supercomputing Facilities, and the authors gratefully acknowledge Facility staff for their help and assistance. The authors also gratefully acknowledge the effort by the anonymous reviewers to improve this manuscript.

\section{References}

[1] Liou KN. Influence of cirrus clouds on weather and climate processes: a global perspective. Mon Weather Rev 1986;114:1167-99.

[2] Lynch DK, Sassen K, O'C Starr D, Stephens G, editors. Cirrus. New York: Oxford University Press; 2002.

[3] Platnick S, King MD, Ackerman SA, Menzel WP, Baum BA, Riedi JC, et al. The MODIS cloud products: algorithms and examples from Terra. IEEE Trans Geosci Remote Sens 2003;41:459-73.

[4] King MD, Platnick S, Yang P, Arnold GT, Gray MA, Riedi JC, et al. Remote sensing of liquid water and ice cloud optical thickness, and effective radius in the Arctic: application of airborne multispectral MAS data. J Atmos Oceanic Technol 2004;21:857-75.

[5] Minnis P, et al. CERES Edition-2 cloud property retrievals using TRMM VIRS and TERRA and AQUA MODIS data-part I: algorithms. IEEE Trans Geosci Remote Sens 2011;49:4374-400.

[6] Wang X, Liou KN, Ou SC, Mace GG, Deng M. Remote sensing of cirrus cloud vertical size profile using MODIS data. J Geophys Res 2009;114:D09205. http://dx.doi.org/10.1029/2008JD011327.

[7] Huang HL, Yang P, Wei H, Baum BA, Hu YX, Atonelli P, et al. Inference of ice cloud properties from high-spectral resolution 
infrared observations. IEEE Trans Geosci Remote Sens 2004:42: $842-53$.

[8] Wendisch M, Yang P, Pilewskie P. Effects of ice crystal habit on the thermal infrared radiative properties and forcing of cirrus clouds. J Geophys Res 2007;112:D08201. http://dx.doi.org/10.1029/200 6JD007899.

[9] Edwards JM, Havemann S, Thelen JC, Baran AJ. A new parameterization for the radiative properties of ice crystals: comparison with existing schemes and impact in a GCM. Atmos Res 2007;83:19-34.

[10] Xie Y, Yang P, Liou KN, Minnis P, Duda DP. Parameterization of contrail radiative properties for climate studies. Geophys Res Lett 2012;39:L00F02. http://dx.doi.org/10.1029/2012GL054043.

[11] Yi B, Yang P, Liou KN, Minnis P, Penner JE. Simulation of the global contrail radiative forcing: a sensitivity analysis. Geophys Res Lett 2012;39:L00F03. http://dx.doi.org/10.1029/2012GL054042.

[12] Jacobowitz H. A method for computing the transfer of solar radiation through clouds of hexagonal ice crystals. J Quant Spectrosc Radiat Transf 1971;11:691-5.

[13] Wendling P, Wendling R, Weickmann HK. Scattering of solar radiation by hexagonal ice crystals. Appl Opt 1979;18:2663-71.

[14] Cai Q, Liou KN. Polarized light scattering by hexagonal ice crystals: theory. Appl Opt 1982;21:3569-80.

[15] Takano Y, Liou KN. Solar radiative transfer in cirrus clouds. Part I: single-scattering and optical properties of hexagonal ice crystals. J Atmos Sci 1989;46:3-19.

[16] Macke A. Scattering of light by polyhedral ice crystals. Appl Opt 1993;32:2780-8.

[17] Muinonen K, Nousiainen T, Fast P, Lumme K, Peltoniemi JI. Light scattering by Gaussian random particles: ray optics approximation. J Quant Spectrosc Radiat Transf 1996;55:577-601.

[18] Nousiainen T, McFarquhar GM. Light scattering by quasi-spherical ice crystals. J Atmos Sci 2004;18:2229-48.

[19] Um J, McFarquhar GM. Single-scattering properties of aggregates of bullet rosettes in cirrus. J Appl Meteor Climatol 2007;46:757-75.

[20] Muinonen K. Scattering of light by crystals: a modified Kirchhoff approximation. Appl Opt 1989;28:3044-50.

[21] Yang P, Liou KN. Geometric-optics-integral-equation method for light scattering by nonspherical ice crystals. Appl Opt 1996;35: 6568-84.

[22] Yang P, Liou KN. Light scattering by hexagonal ice crystals: solution by ray-by-ray integration algorithm. J Opt Soc Am A 1997;14: 2278-89.

[23] Popov AA. New method for calculating the characteristics of light scattering by spatially oriented atmospheric crystals. Proc SPIE 1996;2822:186-94.

[24] Borovoi AG, Grishin IA. Scattering matrices for large ice crystal particles. J Opt Soc Am A 2003;20:2071-80.

[25] Bi L, Yang P, Kattawar GW, Hu Y, Baum BA. Scattering and absorption of light by ice particles: solution by a new physical-geometric optics hybrid method. J Quant Spectrosc Radiat Transf 2011;112:1492-508.

[26] Masuda K, Kshimoto H, Mano Y. Efficient method of computing a geometric optics integral for light scattering by nonspherical particles. Pap Meteorol Geophys 2012;63:15-9.

[27] Deschamps GA. Ray techniques in electromagnetics. Proc. IEEE 1972;60:1022-35.

[28] Mishchenko MI, Tishkovets VP, Travis LD, Cairns B, Dlugach JM, Liu $\mathrm{L}$, et al. Electromagnetic scattering by a morphologically complex object: fundamental concepts and common misconceptions. J Quant Spectrosc Radiat Transf 2011;112:671-92.

[29] Yang P, Liou KN. Light scattering by hexagonal ice crystals: comparison of finite-difference time domain and geometric optics methods. J Opt Soc Am A 1995;12:162-76.

[30] Yee SK. Numerical solution of initial boundary value problems involving Maxwell's equations in isotropic media. IEEE Trans Antennas Propag 1966;14:302-7.

[31] Yang P, Liou KN. Finite-difference time domain method for light scattering by small ice crystals in three-dimensional space. J Opt Soc Am A 1996;13:2072-85.

[32] Sun W, Fu O Chen Z. Finite-difference time-domain solution of light scattering by dielectric particles with perfectly matched layer absorbing boundary conditions. Appl Opt 1999;38:3141-51.

[33] Ishimoto H, Masuda K, Mano Y, Orikasa N, Uchiyama A. Irregularly shaped ice aggregates in optical modeling of convectively generated ice clouds. J Quant Spectrosc Radiat Transf 2012;113:632-43.

[34] Liu QH. The PSTD algorithm: a time-domain method requiring only two cells per wavelength. Microw Opt Technol Lett 1997;15:158-65.

[35] Liu C, Panetta RL, Yang P. Application of the pseudo-spectral time domain method to compute particle single-scattering properties for size parameters up to 200. J Quant Spectrosc Radiat Transf 2012:113: $1728-40$.

[36] Liu C, Bi L, Panetta RL, Yang P, Yurkin MA. Comparison between the pseudo-spectral time domain method and the discrete dipole approximation for light scattering simulations. Opt Express 2012;20: 16763-76.

[37] Purcell EM, Pennypacker CR. Scattering and absorption of light by nonspherical dielectric grains. Astrophys J 1973;186:705-14.

[38] Draine BT. The discrete-dipole approximation and its application to interstellar graphite grains. Astrophys J 1988;333:848-72.

[39] Goedecke GH, O'Brien SG. Scattering by irregular inhomogeneous particles via the digitized Green's function algorithm. Appl Opt $1988 ; 27: 2431-8$.

[40] Yurkin MA, Maltsev VP, Hoekstra AG. The discrete dipole approximation for simulation of light scattering by particles much larger than the wavelength. J Quant Spectrosc Radiat Transf 2007;106: 546-57.

[41] Waterman PC. Matrix formulation of electromagnetic scattering. Proc IEEE 1965;53:805-12.

[42] Waterman PC. Symmetry, unitarity, and geometry in electromagnetic scattering. Phys Rev D 1971;3:825-39.

[43] Mishchenko MI, Travis LD, Mackowski DW. T-matrix computations of light scattering by nonspherical particles: a review. J Quant Spectrosc Radiat Transf 1996;55:535-75.

[44] Doicu A, Eremin Y, Wriedt T. Acoustic and Electromagnetic Scattering Analysis Using Discrete Sources. New York: Academic Press; 2000.

[45] Baran AJ, Yang P, Havemann S. Calculation of the single-scattering properties of randomly oriented hexagonal ice columns: a comparison of the T-matrix and the finite-difference time-domain methods. Appl Opt 2001;40:4376-86.

[46] Mishchenko MI, Macke A. How big should hexagonal ice crystals be to produce halos? Appl Opt 1999;38:1626-9.

[47] Johnson BR. Invariant imbedding T-matrix approach to electromagnetic scattering. Appl Opt 1988;27:4861-7.

[48] Bi L, Yang P, Kattawar GW, Mishchenko MI. Efficient implementation of the invariant imbedding T-matrix method and the separation of variables method applied to large nonspherical inhomogeneous particles. J Quant Spectrosc Radiat Transf 2013;116:169-83.

[49] Bi L, Yang P, Kattawar GW, Mishchenko MI. A numerical combination of extended boundary condition and invariant imbedding method applied to light scattering by large spheroids and cylinders. J Quant Spectrosc Radiat Transf 2013;123:17-22.

[50] Bi L, Yang P. Accurate simulation of the optical properties of atmospheric ice crystals with invariant imbedding T-matrix method. J Quant Spectrosc Radiat Transf 2014;138:36-49.

[51] Bi L, Yang P. Physical-geometric optics hybrid methods for computing the scattering and absorption properties of ice crystals and dust aerosols. In: Kokhanovsky A, editor. Light scattering reviews, 8 Chichester, UK: Springer-Praxis Publishing; 2013 (69-11).

[52] Yang P, Liou KN. Light scattering and absorption by nonspherical ice crystals In: Kokhanovsky A., editor. Light scattering reviews: single and multiple light scattering. Springer-Praxis. p. 31-71.

[53] Fu Q. A new parameterization of an asymmetry factor of cirrus clouds for climate models. J Atmos Sci 2007;64(11):4140. http://dx.doi.org/ 10.1175/2007JAS2289.1.

[54] Mishchenko MI, Macke A. Incorporation of physical optics effects and computation of the Legendre expansion for ray-tracing scattering functions involving $\delta$-function transmission. J Geophys Res 1998;103:1799-805. http://dx.doi.org/10.1029/97JD03121.

[55] Yang P, Bi L, Baum BA, Liou KN, Kattawar GW, Mishchenko MI, et al. Spectrally consistent scattering, absorption, and polarization properties of atmospheric ice crystals at wavelengths from 0.2 to $100 \mu \mathrm{m}$. J Atmos Sci 2013;70:330-47.

[56] Warren SG, Brandt RE. Optical constants of ice from the ultraviolet to the microwave: a revised compilation. J Geophys Res 2008;113: D14220. http://dx.doi.org/10.1029/2007JD009744.

[57] Baum BA, Yang P, Heymsfield AJ, Schmitt CG, Xie Y, Bansemer A, et al. Improvements in shortwave bulk scattering and absorption models for the remote sensing of ice clouds. J Appl Meteorol Clim 2011;50:1037-56.

[58] Iwabuchi H, Yang P, Liou KN, Minnis P. Physical and optical properties of persistent contrails: climatology and interpretation. J Geophys Res 2012;117:D06215.

[59] Nakajima T, King MD. Determination of the optical thickness and effective particle radius of clouds from reflected solar radiation measurements. Part 1. Theory. J Atmos Sci 1990;47:1878-93.

[60] Wang C, Yang P, Nasiri SL, Platnick S, Baum BA, Heidinger AK, et al. A fast radiative transfer model for visible through shortwave 
infrared spectral reflectances in clear and cloudy atmospheres. J Quant Spectrosc Radiat Transf 2013;116:122-31.

[61] Wang C, Yang P, Platnick S, Heidinger AK, Baum BA, Greenwald T, et al. Retrieval of ice cloud properties from AIRS and MODIS observations based on a fast high-spectral-resolution radiative transfer model. J Appl Meteorol Clim 2013;52:710-25.

[62] Wang C, Yang P, Platnick S, Heidinger AK, Baum BA, Greenwald T,

et al. Retrieval of ice cloud properties from AIRS and MODIS observations based on a fast high-spectral-resolution radiative transfer model. J Appl Meteorol Clim 2013;52:710-25.

[63] Zhang Z, Yang P, Kattawar GW, Riedi J, Labonnote LC, Baum BA, et al. Influence of ice particle model on satellite ice cloud retrieval: lessons learned from MODIS and POLDER cloud product comparison. Atmos Chem Phys 2009;9:7115-29.

[64] Iacono MJ, Delamere JS, Mlawer EJ, Shephard MW, Clough SA Collins WD. Radiative forcing by long-lived greenhouse gases: calculations with the AER radiative transfer models. J Geophys Res 2008;113:D13103. http://dx.doi.org/10.1029/2008JD009944.

[65] Yi B, Yang P, Baum BA, L'Ecuyer T, Oreopoulos L, Mlawer EJ, et al. Influence of ice particle surface roughening on the global cloud radiative effect. J Atmos Sci 2013;70:2794-807. http://dx.doi.org/ 10.1175/JAS-D-13-020.1

[66] van Diedenhoven B, Fridlind AM, Ackerman AS, Cairns B. Evaluation of hydrometeor phase and ice properties in cloud-resolving model simulations of tropical deep convection using radiance and polarization measurements. J Atmos Sci 2012;69(11):3290-314. http://dx.doi.org/ 10.1175/JAS-D-11-0314.1.

[67] Yang P, Zhang L, Hong G, Nasiri SL, Baum BA, Huang HL, et al. Differences between collection 4 and 5 MODIS ice cloud optical/ microphysical products and their impact on radiative forcing simulations. IEEE Trans Geosci Remote Sens 2007;45:2886-99.

[68] Yang P, Hong G, Kattawar GW, Minnis P. Uncertainties associated with the surface texture of ice particles in satellite based retrieval of cirrus clouds: part II-effect of particle surface roughness on retrieved cloud optical thickness and effective particle size. IEEE Trans Geosci Remote Sens 2008;46:1948-57.

[69] Van Diedenhoven B, Ackerman AS, Cairns B, Fridlind AM. A flexible parameterization for shortwave optical properties of ice crystals. J. Atmos. Sci., http://dx.doi.org/10.1175/JAS-D-13-0205.1, in press. 